\title{
Analyses of Catharanthus roseus and Arabidopsis thaliana WRKY transcription factors reveal involvement in jasmonate signaling
}

\author{
Craig Schluttenhofer', Sitakanta Pattanaik², Barunava Patra ${ }^{2}$ and Ling Yuan ${ }^{1,2^{*}}$
}

\begin{abstract}
Background: To combat infection to biotic stress plants elicit the biosynthesis of numerous natural products, many of which are valuable pharmaceutical compounds. Jasmonate is a central regulator of defense response to pathogens and accumulation of specialized metabolites. Catharanthus roseus produces a large number of terpenoid indole alkaloids (TIAs) and is an excellent model for understanding the regulation of this class of valuable compounds. Recent work illustrates a possible role for the Catharanthus WRKY transcription factors (TFs) in regulating TIA biosynthesis. In Arabidopsis and other plants, the WRKY TF family is also shown to play important role in controlling tolerance to biotic and abiotic stresses, as well as secondary metabolism.

Results: Here, we describe the WRKY TF families in response to jasmonate in Arabidopsis and Catharanthus. Publically available Arabidopsis microarrays revealed at least 30\% (22 of 72) of WRKY TFs respond to jasmonate treatments. Microarray analysis identified at least six jasmonate responsive Arabidopsis WRKY genes (AtWRKY7, AtWRKY20, AtWRKY26, AtWRKY45, AtWRKY48, and AtWRKY72) that have not been previously reported. The Catharanthus WRKY TF family is comprised of at least 48 members. Phylogenetic clustering reveals 11 group I, 32 group II, and 5 group III WRKY TFs. Furthermore, we found that at least 25\% (12 of 48) were jasmonate responsive, and $75 \%$ (9 of 12) of the jasmonate responsive CrWRKYs are orthologs of AtWRKYs known to be regulated by jasmonate.

Conclusion: Overall, the CrWRKY family, ascertained from transcriptome sequences, contains approximately $75 \%$ of the number of WRKYs found in other sequenced asterid species (pepper, tomato, potato, and bladderwort). Microarray and transcriptomic data indicate that expression of WRKY TFs in Arabidopsis and Catharanthus are under tight spatio-temporal and developmental control, and potentially have a significant role in jasmonate signaling. Profiling of CrWRKY expression in response to jasmonate treatment revealed potential associations with secondary metabolism. This study provides a foundation for further characterization of WRKY TFs in jasmonate responses and regulation of natural product biosynthesis.
\end{abstract}

Keywords: Catharanthus roseus, Terpenoid indole alkaloid, Transcriptome, Secondary metabolism, WRKY transcription factors

\footnotetext{
* Correspondence: lyuan3@uky.edu

${ }^{1}$ Department of Plant and Soil Science, University of Kentucky, Lexington, KY 40546, USA

${ }^{2}$ Kentucky Tobacco Research and Development Center, University of Kentucky, Lexington, KY 40546, USA
}

\section{() Biomed Central}

(c) 2014 Schluttenhofer et al.; licensee BioMed Central Ltd. This is an Open Access article distributed under the terms of the Creative Commons Attribution License (http://creativecommons.org/licenses/by/2.0), which permits unrestricted use, distribution, and reproduction in any medium, provided the original work is properly credited. The Creative Commons Public Domain Dedication waiver (http://creativecommons.org/publicdomain/zero/1.0/) applies to the data made available in this article, unless otherwise stated. 


\section{Background}

Secondary metabolites (a.k.a natural products or specialized metabolites), are compounds synthesized by plants for attracting pollinators [1], inter-plant communication [2], and defense [3]. The plethora of natural products synthesized by plants also provides many valuable pharmaceutical compounds. Catharanthus roseus (L.) G. Don, commonly called Madagascar periwinkle or annual vinca, belongs to the Apocynaceae family and synthesizes over 130 terpene indole alkaloids (TIAs) including the pharmaceutically valuable vinblastine and vincristine. Vinblastine and vincristine provide antineoplastic compounds effective in the treatment of several types of cancer [4]. The biosynthesis of these compounds, along with other TIAs, is regulated by UV light $[5,6]$, fungal elicitors [7], wounding $[8,9]$, drought [10], cold [11,12] and salt stress [12]. A principal elicitor of TIA production in Catharanthus, as well as natural products in many other medicinal species, is the phytohormone jasmonate which functions in plant defense signaling to protect the plant from biotic stresses $[3,13]$.

Transcription factors (TFs) play a critical role in responding to jasmonate to elicit the synthesis of TIAs in Catharanthus [14-16]. Negative regulators also mediate jasmonate signaling of the TIA pathway in Catharanthus $[17,18]$. In plants, WRKY TFs are critical regulators of response to biotic and abiotic stress. WRKY TFs have been attributed to tolerance of drought [19], salt [20], nutrient deficiency [21], osmotic [22], cold [23], heat [24], oxidative [25], wounding [26], pathogens [27], and UV-B stresses [28]. The WRKY TF family is primarily a plant specific family with the exception of several examples in protozoa [29]. The WRKY domain is a 60 to 70 amino acid long DNA binding domain that recognizes the W-box (TTGACC/T); however, recent studies suggest this cis-element may be more degenerate and other components are involved for WRKY binding to DNA in response to a specific stimulus [30,31]. The N-terminal portion of the WRKY domain is characterized by a highly conserved WRKYGQK motif whereas the $\mathrm{C}$-terminal region of the domain contains either a $\mathrm{Cys}_{2}-\mathrm{His}_{2}$ or $\mathrm{Cys}_{2}$-His-Cys zinc-finger [32]. WRKY TFs are distinguished by the presence of one or two WRKY domains. Group I WRKYs typically contain two WRKY domains whereas group II and group III members only contain one WRKY domain [32]. Up to five subgroups (IIa, IIb, IIc, IId, and IIe) are recognized in the group II WRKY TFs [32].

In Arabidopsis, WRKY TFs are well established in salicylic acid (SA) and defense signaling pathways [33-35]. The majority of Arabidopsis WRKY TFs are induced by treatment with SA [33]. However, the importance of WRKY TFs in JA signaling network is relatively less studied. Li et al. [36] identified AtWRKY70 as a positive regulator of SA signaling and negative regulator of jasmonate signaling. Mutation of AtWRKY33 enhances susceptibility to necrotrophic pathogens by up-regulating JAZ proteins, repressors of jasmonate signaling [27,37]. Jasmonate positively regulates AtWRKY18 and AtWRKY4O which also negatively regulate abscisic acid (ABA) response [38]. AtWRKY6, AtWRKY8, AtWRKY11, AtWRKY17, AtWRKY25, AtWRKY28, AtWRKY38, AtWRKY60, AtWRKY62, and AtWRKY70 are also differentially expressed by jasmonates to regulate plant defense [22,39-45].

Over the last several years, WRKY TFs have emerged as a key family in the induction of natural product biosynthesis [46,47]. CjWRKY1, from Coptis japonica, regulates the production of the benzylisoquinoline alkaloid, berberine [48]. Cotton (Gossypium arboreum) GaWRKY1 affects the biosynthesis of the sesquiterpene, gossypol [49]. Multiple biosynthetic genes for the sesquiterpene lactone, artemisinin, valuable as an anti-malaria drug, are regulated by Artemisia annua WRKY1 [50]. Hevea brasiliensis WRKY1 is present in the latex of mechanically wounded (tapped) trees suggesting involvement in rubber latex synthesis [51]. The Taxus chinensis WRKY1 was found to regulate the expression of 10-DEACETYLBACCATIN III-10 B-OACETYL TRANSFERASE (DBAT), a gene encoding a key enzyme catalyzing a rate limiting step in the biosynthesis of the anticancer terpene, paclitaxel [47]. In Arabidopsis, camalexin biosynthesis is mis-regulated in wrky33 mutant [52]. Over-expression in Arabidopsis of Panax quinquefolius WRKY1, a jasmonate responsive WRKY from American ginseng, is found to enhance expression of genes related to drought, salt, and disease resistance, leading to improvement of seedling survival to drought and salt stress, in addition to regulating the expression of genes related to triterpene biosynthesis [53]. In Catharanthus, CrWRKY1 has been demonstrated to respond to jasmonate, ethylene, and gibberellin signaling to regulate TIA production [46]. Over-expression of CrWRKY1 increased the production of serpentine while simultaneously decreasing catharanthine accumulation, suggesting this WRKY may function in governing gene expression that specifically directs the flow of metabolites to synthesize TIAs in Catharanthus roots.

Identification of jasmonate responsive WRKY will thus provide useful information on plant defense and natural product regulatory networks. Understanding the number and types of WRKY TFs present in Catharanthus will provide a clearer picture on the regulation of TIAs by this important TF family. Here, we present jasmonate responsive WRKYs from Arabidopsis and Catharanthus. First, we analyzed Arabidopsis microarray data to help identify the involvement of the WRKY TFs in jasmonate signaling. We then used the medicinal plant transcriptome data to identify the Catharanthus family of WRKY TFs. Expression data from Catharanthus revealed the induction of multiple WRKY transcripts by methyl jasmonate (MeJA) treatment. Seventy-five percent of the jasmonate responsive 
CrWRKYs are orthologs of AtWRKYs known to be regulated by jasmonate. MeJA-induced WRKYs provide potential candidates for further regulation of TIA accumulation in Catharanthus. The identification of orthologs for WRKY TFs known to be involved in specialized metabolism in other plant species indicates the possible involvement of additional WRKY TFs in regulation of TIA production in Catharanthus.

\section{Results and discussion}

\section{WRKY TFs are involved in Jasmonate signaling}

The role of WRKY TF family in SA signaling and plant defense is well established and has been systematically analyzed in Arabidopsis, but remains less clear for jasmonate signaling. Jasmonate is a key phytohormone regulating the production of specialized metabolites in many plant species, including Catharanthus. While Arabidopsis does not synthesize TIAs as found in Catharanthus, studying AtWRKYs can answer several important questions. First we wanted to determine whether the WRKY family is important for regulating jasmonate signaling in a model species, such as Arabidopsis. Second, we wanted to elucidate jasmonate responsive AtWRKYs to aid the identification of
CrWRKY orthologs with potentially conserved regulatory functions. Comparison of orthologous jasmonate responsive WRKYs from Arabidopsis and Catharanthus will identify WRKYs that are potentially involved in modulating jasmonate signaling, and in turn identify candidates that regulate TIA production.

To clearly establish the role of WRKY TFs in jasmonate signaling we first identified jasmonate responsive WRKYs in the model plant Arabidopsis. To ascertain jasmonate responsive Arabidopsis WRKY TFs we used publically available microarray datasets (Table 1). The ATH1 Affymetrix arrays used contain probes identifying 85\% (61 of the 72) of Arabidopsis WRKY TFs (Additional file 1: Table S1). From five datasets, we identified 39 AtWRKY TFs that significantly change in response to jasmonate treatment (Additional file 2: Table S2). Of the 39 jasmonate responsive AtWRKY genes, 22 were differentially expressed in at least two jasmonate treated datasets. AtWRKY6, AtWRKY18, AtWRKY45, and AtWRKY53 were differentially expressed in three jasmonate treated datasets. Expression of AtWRKY7, AtWRKY69 and AtWRKY75 were significantly changed in response to jasmonate in four datasets. AtWRKY40 and AtWRKY47 expression were

Table 1 The Arabidopsis WRKY transcription factors differentially expressed in response to jasmonate treatment in five experiments before and after the application of the Benjamini-Hochberg false discovery rate

\begin{tabular}{|c|c|c|c|c|c|c|c|c|}
\hline \multirow[b]{2}{*}{ Dataset } & \multirow[b]{2}{*}{ Source } & \multirow[b]{2}{*}{ Samples used } & \multicolumn{3}{|c|}{ Genes after Two-Way ANOVA $(P=0.05)$} & \multicolumn{3}{|c|}{$\begin{array}{l}\text { Genes after Two-Way ANOVA } \\
(\mathrm{P}=0.05) \text { and B-H FDR }\end{array}$} \\
\hline & & & $\begin{array}{l}\text { No. } \\
\text { genes }\end{array}$ & $\begin{array}{l}\text { No. } \\
\text { WRKY } \\
\text { genes }\end{array}$ & WRKYs & $\begin{array}{l}\text { No. } \\
\text { genes }\end{array}$ & $\begin{array}{l}\text { No. } \\
\text { WRKY } \\
\text { genes }\end{array}$ & WRKYs \\
\hline E-ATMX-13 & EMBL & $\begin{array}{l}\text { MeJA treated } \\
\text { timecourse }(0.5, \\
2 \text {, and } 6 \mathrm{hr}) \text { in cell } \\
\text { suspension cultures }\end{array}$ & 2819 & 11 & $\begin{array}{l}\text { WRKY6, WRKY9, WRKY15, } \\
\text { WRKY18, WRKY25, WRKY26, } \\
\text { WRKY39, WRKY40, WRKY47, } \\
\text { WRKY54, WRKY69 }\end{array}$ & 116 & none & none \\
\hline E-GEOD-28600 & EMBL & $\begin{array}{l}J A \text { and } J A+A B A \\
\text { treated ( } 3 \text { and } 24 \mathrm{hr}) \\
\text { T87 cell cultures }\end{array}$ & 5279 & 21 & $\begin{array}{l}\text { WRKY1, WRKY6, WRKY7, } \\
\text { WRKY16, WRKY21, WRKY35, } \\
\text { WRKY36, WRKY38, WRKY40, } \\
\text { WRKY43, WRKY45, WRKYY47, } \\
\text { WRKY52, WRKY53, WRKY54, } \\
\text { WRKY67, WRKY69, WRKY70, } \\
\text { WRKY71, WRKY72, WRKY75 }\end{array}$ & 533 & 3 & WRKY7, WRKY38, WRKY70 \\
\hline E-MEXP-883 & EMBL & $\begin{array}{l}\text { MeJA treated }(6 \mathrm{hr}) \\
\text { WT and myc } 2 \text { plants }\end{array}$ & 3348 & 14 & $\begin{array}{l}\text { WRKY6, WRKY7, WRKY11, } \\
\text { WRKY18, WRKY20, WRKYY23, } \\
\text { WRKY26, WRKY33, WRKY39, } \\
\text { WRKY40, WRKY45, WRKY47, } \\
\text { WRKY69, WRKY75 }\end{array}$ & 568 & 4 & $\begin{array}{l}\text { WRKY26, WRKY33, WRKY40, } \\
\text { WRKY45 }\end{array}$ \\
\hline GSE21762 & $\mathrm{NCBI}$ & $\begin{array}{l}J A \text { treated } W T \text { and } \\
\text { coil seedlings }\end{array}$ & 3743 & 16 & $\begin{array}{l}\text { WRKY3, WRKY7, WRKY17, } \\
\text { WRKY22, WRKY25, WRKYY1, } \\
\text { WRKY40, WRKY46, WRKYY47, } \\
\text { WRKY52, WRKY53, WRKY60, } \\
\text { WRKY70, WRKY72, WRKY74, } \\
\text { WRKY75 }\end{array}$ & 175 & 1 & WRKY72 \\
\hline ME00337 & TIAR & $\begin{array}{l}\text { MeJA treated }(0.5,1, \\
3 \mathrm{hr}) \text { time course on } \\
\text { WT seedlings }\end{array}$ & 4796 & 15 & $\begin{array}{l}\text { WRKY3, WRKY7, WRKY18, } \\
\text { WRKY20, WRKY21, WRKYY23, } \\
\text { WRKY38, WRKY40, WRKY45, } \\
\text { WRKY47, WRKY48, WRKY53, } \\
\text { WRKY60, WRKY69, WRKY75 }\end{array}$ & 950 & 6 & $\begin{array}{l}\text { WRKY7, WRKY18, WRKY20, } \\
\text { WRKY40, WRKY45, WRKY48 }\end{array}$ \\
\hline
\end{tabular}


significantly changed in all five datasets. Notably, AtWRKY47, AtWRKY69, and AtWRKY75 expression were significantly differentially regulated to jasmonate treatment in four or more microarray experiments, but did not survive application of the Benjamini-Hochberg false discovery (B-H FDR) in any dataset. Arabidopsis WRKY6, WRKY11, WRKY17, WRKY25, WRKY46, and WRKY53, which have known roles in jasmonate signaling, were identified as being differentially expressed, but did not survive the B-H FDR in any dataset (Additional file 2: Table S2). While the B-H FDR is less conservative than other procedures (e.g. Bonferroni correction), genes on the upper end of significant p-values (genes with small fold changes) may not be easily detected even if the response is consistent. This becomes apparent by the large reduction in significant genes after B-H FDR (Table 1). In total, eleven WRKYs survived the B-H FDR in at least one dataset indicating these are jasmonate responsive. We identified five WRKY TFs previously reported to be jasmonate responsive $[27,36,38,45]$. Additionally, we identified at least six Arabidopsis WRKY TFs (AtWRKY7, AtWRKY20, AtWRKY26, AtWRKY45, AtWRKY48, and $A t W R K Y 72)$ previously unreported to have a role in jasmonate response (Table 1 and Additional file 2: Table S2). Expression of AtWRKY7, AtWRKY40, and AtWRKY45 changed significantly and survived the B-H FDR in two datasets. For these eleven AtWRKYs, the change in expression in response to jasmonate treatment was small, around 2.5 fold (Additional file 3: Table S3A-E). AtWRKY40 displayed the greatest change in expression, with a 9-fold induction of transcripts after $1 \mathrm{hr}$ of treatment with MeJA. Collectively, at least $18 \%$ of AtWRKY (11 of 61), up to $64 \%$ (39 of 61) or more, were jasmonate responsive WRKYs based on the microarrays analyzed. Overall, at least 30\% (22 of 72) of AtWRKY TFs play a role in jasmonate response indicating the importance of this family in the jasmonate signaling network. Further experiments may eventually reveal that upwards of 50\% (39 of 72) of WRKYs are involved in Arabidopsis jasmonate response (Additional file 2: Table S2).

The small overlap between WRKY genes differentially expressed in response to jasmonate treatment in the microarray experiments suggested a tight developmental and/or spatiotemporal regulation in Arabidopsis. Two-way ANOVAs analyzing expression in response to time, as well as its combined effect with jasmonate treatment, further indicated AtWRKY regulation is temporally dependent (Additional file 4: Table S4A-C). The expression of 20 AtWRKY genes was time dependent (Additional file 4: Table S4A). Jasmonate treatment was found to regulate the expression of AtWRKY38 and AtWRKY7O in a time dependent manner. The genetic background of jasmonate signaling pathway mutants had less effect on WRKY gene expression. CORONATINE INSENSITIVE 1 (COI1) has been established as a jasmonate receptor [54,55]. AtWRKY72 was the only WRKY family member found to be regulated in a COI1-dependent manner when the B-H FDR was applied (Additional file 4: Table S4B). No WRKYs were found to be dependent on MYC2 (Additional file 4: Table S4C), a major transcriptional regulator of jasmonate signaling $[56,57]$. These results indicate that response of Arabidopsis WRKY TFs to jasmonate treatment is highly dependent upon tissue, timing and culture conditions, and likely occurs through several major pathways. Furthermore, WRKY TFs may be important COI1-independent regulators of jasmonate response.

Unsupervised agglomerative hierarchical clustering analysis of AtWRKY TFs was performed to identify similar patterns of gene expression which may indicate related functions [58]. Gene expression of AtWRKYs formed two major clusters. Clustering of experiments revealed more similarities within an experiment than by jasmonate treatment (Additional file 5: Figure S1). Additionally, the two major clusters separated those experiments in which the sampled tissues were from either plants or cell cultures. These findings further support AtWRKY gene expression in response to jasmonate as highly dependent on culture conditions and environment. The two major clusters were further subdivided into two or three clusters. Jasmonate responsive WRKYs previously annotated or identified by our microarray analysis primarily occurred in cluster one and all of the three sub-clusters. The distribution of jasmonate responsive AtWRKYs indicates at least two major pathways for the regulation of AtWRKY gene expression. Interestingly, the only WRKY identified by microarray analysis to be COI1-depenedent, AtWRKY72, occurred in cluster $2 \mathrm{~b}$, distinct from expression patterns of other jasmonate responsive WRKYs. These data further suggest that there are complex tissue and environmental controls over jasmonate responses that likely occurs through several major pathways. These findings from Arabidopsis provide foundational information about the involvement of WRKY TFs in jasmonate response and for exploiting these factors in genetic engineering of transcriptional regulatory networks for natural product production.

\section{Identification of Catharanthus WRKY TFs}

Previously we identified a MeJA responsive group III type WRKY TF, CrWRKY1, as important for the regulation of TIA in Catharanthus [46]. Furthermore, the Arabidopsis data indicates the AtWRKY family as important for regulating jasmonate response. Jasmonate responsive CrWRKY TF may be important for regulating the production of valuable TIAs. Elucidation of CrWRKYs regulating specialized metabolite production will be valuable for future genetic engineering projects to increase production of pharmaceutically valuable TIAs. As the first step to identify important CrWRKY regulators of specialized metabolism 
we sought to identify all WRKY family members in Catharanthus. The recent release of 14 medicinal plants Illumina sequenced transcriptomes by the Medicinal Plant Genomic Resource (MPGR), including Catharanthus, provides the opportunity to identify many CrWRKY TFs [59]. To identify CrWRKYs, we first downloaded all protein sequences from MPGR and isolated a single protein sequence for each locus. We assumed the individual copy of each locus reflects the total number of functional genes within the genome. While this method may include potential errors, such as RNA-sequencing artifacts, establishing single copies of genes allows the identification of WRKY family members and approximate family size. Due to possible variations in splicing and/or incomplete splicing of introns we searched contig assemblies with the longest predicted protein sequence for each gene in the MPGR database. All CrWRKY proteins identified from the MPGR database, described below, were manually verified to contain a WRKY domain. In several cases (CrWRKY8, CrWRKY13, CrWRKY17, CrWRKY21, CrWRKY34, CrWRKY37, and CrWRKY47), alignment results among Catharanthus contigs for a locus and the closest matching AtWRKY TF, were utilized to remove a conserved intron following the WRKYGQK consensus sequence or correct a frame shift, to generate a full WKRY domain sequence. As single base pair insertion in CrWRKY8 was not clear by aligning other copies of this contig, the region spanning the insertion was cloned for verification.

Searching for the established invariant consensus sequences WRKYGQK and known alternative WRKYGKK, WRKYGEK, and WRKYGSK consensus sequences from the list of proteins, duplicate results were eliminated and 46 putative WRKY TFs were identified (Additional file 6: Table S5). Comparatively, MPGR annotated 47 potential CrWRKY TF encoding genes. However, only 35 WRKYs overlapped between manual searches for the consensus motifs and the MPGR annotated datasets.

To further validate the number of WRKY TFs a list of the single longest predicted proteins for each locus was submitted to the National Center for Biotechnology Information Conserved Domain Database (NCBI CDD) and the Samuel Roberts Nobel Foundation PlantTFcat (PlantTFcat) server, for protein domain identification [60]. The NCBI CDD identified 52 WRKY domain-containing proteins (Additional file 6: Table S5). Similarly, PlantTFcat (http://plantgrn.noble.org/PlantTFcat/) also identified 52 WRKY domain-containing proteins. The majority of additional proteins identified by NCBI CDD and PlantTFcat as WRKY TFs, had incomplete N-terminal ends of the WRKY domain (CrWRKY11, CrWRKY15, CrWRKY48, and CrWRKY49). One additional predicted WRKY TF, CrWRKY32, contained a WRKYGRK motif. CrWRKY9, which was identified by NCBI CDD, but not PlantTFcat, had an incomplete C-terminal portion of the WRKY domain. Contig Cra15757 was predicted by PlantTFcat to be a WRKY TF. Inspection of this protein sequence did not reveal the presence of a WRKY consensus or zinc finger binding motif. Of the 47 proteins annotated as WRKYs by MPGR, only 40 were found to be true WRKY TFs as identified by NCBI CDD and PlantTFcat. In total, 52 proteins in Catharanthus were predicted as WRKY TFs (Table 2).

Of the 52 possible WRKY TFs from Catharanthus, at least 48 appear to be authentic (Table 2). The MPGR database contained full WRKY domain sequences for 52 domains from 43 TFs. Partial domain sequences were found for nine WRKYs. 3' rapid amplification of cDNA ends (RACE) or 5' RACE was performed to obtain the necessary domain sequence for 5 WRKYs. 3' RACE was performed on CrWRKY9. For CrWRKY11, CrWRKY12, CrWRKY15, and CrWRKY48, 5' RACE was used to obtain the rest of the WRKY domain sequence. Clones could not be found for four genes (CrWRKY49, CrWRKY50, CrWRKY51, and CrWRKY52). Expression data, available from MPGR, revealed these four WRKYs are not present in any of the 23 samples sequenced. To validate the MPGR expression data, quantitative reverse transcription polymerase chain reaction (qRT-PCR) was used to measure the transcript levels of CrWRKY49, CrWRKY50, CrWRKY51, and CrWRKY52. Gene specific transcripts for CrWRKY49, CrWRKY50, CrWRKY51, and CrWRKY52 could not be detected in root, stem, leaf, or whole plant samples. Transcripts for the same four WRKYs could also not be found in $0,1,2$, and 4 hour MeJA-treated samples. This data suggests that these predicted partial WRKY sequences are not in any of our samples, and that they may be either artifacts of RNA-sequencing, temporally regulated, or induced by a factor not present in our growing conditions. WRKY TFs are known to play key roles in plant senescence [42]. However, senescing medicinal plant tissues were not utilized for sequencing in the MPGR. Inclusion of senescing tissues may slightly increase the total WRKY number to more closely reflect fully sequenced plant species. Future investigations with different treatment conditions may detect the expression of CrWRKY49, CrWRKY50, CrWRKY51, and CrWRKY52.

The Catharanthus WRKY family appears to be one of the smallest reported WRKY TF families to date. Only the moss Physcomitrella patens, the lycophyte Selaginella moellendorffii, and Castor bean (Ricinus communis), with 37, 35, and 47 WRKYs respectively, are reported to have fewer WRKY TFs [61-63]. Our results suggest that the Catharanthus WRKY family is similar in size to Cucumis sativus, Fragaria vesca, Jatropha curcas, and Carica papaya with 55, 56, 58 and 66 WRKYs, respectively [64-67]. To further investigate the size of the Catharanthus WRKY family, we identified the WRKY TFs from serpentwood (Rauvolfia serpentina) transcriptome sequences (Table 3) [59]. Serpentwood is closely related to Catharanthus and 
Table 2 A list of Catharanthus WRKY domain containing proteins along with locus number and group number are presented

\begin{tabular}{|c|c|c|}
\hline Catharanthus WRKY & Locus & Group \\
\hline CrWRKY1 & Cra16284 & III \\
\hline CrWRKY2 & Cra549 & I \\
\hline CrWRKY3 & Cra4234 & I \\
\hline CrWRKY4 & Cra5497 & I \\
\hline CrWRKY5 & Cra6088 & I \\
\hline CrWRKY6 & Cra8145 & । \\
\hline CrWRKY7 & Cra9152 & 1 \\
\hline CrWRKY8 & Cra10348 & I \\
\hline CrWRKY9 & Cra11128 & I \\
\hline CrWRKY10 & Cra13321 & । \\
\hline CrWRKY11 & Cra22691 & I \\
\hline CrWRKY12 & Cra43671 & I \\
\hline CrWRKY13 & Cra1311 & Ila \\
\hline CrWRKY14 & Cra13263 & Ila \\
\hline CrWRKY15 & Cra54213 & Ila \\
\hline CrWRKY16 & Cra2068 & $\| \mathrm{b}$ \\
\hline CrWRKY17 & Cra3503 & $\| \mathrm{b}$ \\
\hline CrWRKY18 & Cra18915 & $11 \mathrm{~b}$ \\
\hline CrWRKY19 & Cra19580 & $1 \mathrm{lb}$ \\
\hline CrWRKY20 & Cra22725 & $11 \mathrm{~b}$ \\
\hline CrWRKY21 & Cra2271 & Ilc \\
\hline CrWRKY22 & Cra2950 & \|lc \\
\hline CrWRKY23 & Cra6519 & Ilc \\
\hline CrWRKY24 & Cra8670 & \|c \\
\hline CrWRKY25 & Cra9369 & Ilc \\
\hline CrWRKY26 & Cra19330 & \|lc \\
\hline CrWRKY27 & Cra22395 & IIC \\
\hline CrWRKY28 & Cra24943 & \|c \\
\hline CrWRKY29 & Cra28262 & Ilc \\
\hline CrWRKY30 & Cra37309 & \|c \\
\hline CrWRKY31 & Cra43896 & Ilc \\
\hline CrWRKY32 & Cra102390 & Ilc \\
\hline CrWRKY33 & Cra105225 & IIC \\
\hline CrWRKY34 & Cra1702 & $\| d$ \\
\hline CrWRKY35 & Cra3760 & Ild \\
\hline CrWRKY36 & Cra7867 & $\| d$ \\
\hline CrWRKY37 & Cra17347 & Ild \\
\hline CrWRKY38 & Cra11684 & lle \\
\hline CrWRKY39 & Cra16307 & lle \\
\hline CrWRKY40 & Cra19395 & lle \\
\hline CrWRKY41 & Cra20290 & lle \\
\hline
\end{tabular}

Table 2 A list of Catharanthus WRKY domain containing proteins along with locus number and group number are presented (Continued)

$\begin{array}{lcc}\text { CrWRKY42 } & \text { Cra21821 } & \text { Ile } \\ \text { CrWRKY43 } & \text { Cra23742 } & \text { Ile } \\ \text { CrWRKY44 } & \text { Cra30069 } & \text { Ile } \\ \text { CrWRKY45 } & \text { Cra3799 } & \text { III } \\ \text { CrWRKY46 } & \text { Cra5093 } & \text { III } \\ \text { CrWRKY47 } & \text { Cra18989 } & \text { II } \\ \text { CrWRKY48 } & \text { Cra24719 } & \text { III } \\ \text { CrWRKY49 } & \text { Cra55720 } & \\ \text { CrWRKY50 } & \text { Cra56567 } & \\ \text { CrWRKY51 } & \text { Cra65443 } & \\ \text { CrWRKY52 } & \text { Cra70197 }\end{array}$

also produces pharmaceutically valuable TIAs (Additional file 7: Figure S2). We found 54 serpentwood TFs, a number close to the 52 WRKYs identified in Catharanthus. The number of WRKYs belonging to each subgroup was also similar between these two species (Additional file 8: Figure S3). However, as both serpentwood and Catharanthus WRKYs were identified from transcriptome data, the actual size of the families may be larger. To address this possibility, we identified WRKY families from tomato (Solanum lycopersicum) [68], potato (Solanum tuberosum) [69], pepper (Capsicum annuum) [70], and bladderwort (Utricularia gibba) [71], all species of which have complete genome sequence available (Table 3). We identified 81 WRKY TFs in tomato (Table 3, Additional file 9: Figure S4), as previously reported [72]. Bladderwort, pepper, and potato each contained 65,66 , and 75 complete WRKY TFs, respectively (Table 3 , Additional file 8: Figure S3 and Additional file 9: Figure S4). These data suggest the ancestor of the Gentianales (Catharanthus and serpentwood), Lamiales (bladderwort), and Solanales (pepper, potato and tomato) likely contained around 65 WRKY TFs. Therefore, we conclude that greater than 75\% (52 out of 65) of Catharanthus and serpentwood WRKY TFs were identified from transcriptome data. Together, the six asterid species contained a similar number of WRKY TFs as found in the Brassicales, Arabidopsis and papaya $[66,73]$. These data, combined with that from other WRKY families [64-67], suggests that Brachypodium distachyon (86 WRKYs), Oryza sativa ssp. japonica (105 WRKYs), Populus trichocarpa (104. WRKYs), and Zea mays (119 WRKYs) may contain atypically large WRKY families $[29,33,66,74,75]$ compared to other angiosperms. Arabidopsis and rice both contain expansions in the group III WRKY subfamily $[29,73]$, whereas an expansion of group IIe occurs in potato and tomato [72] (Table 3). We did not find any evidence for subfamily expansions in Catharanthus or serpentwood (Table 3; Figure 1). 
Table 3 The distribution of WRKY TFs from nine plant species

\begin{tabular}{|c|c|c|c|c|c|c|c|c|c|c|}
\hline Species & $\begin{array}{c}\text { Complete WRKY } \\
\text { TFs }\end{array}$ & $\begin{array}{c}\text { Partial WRKY } \\
\text { TFs }\end{array}$ & $\begin{array}{c}\text { Group } \\
I\end{array}$ & $\begin{array}{c}\text { Group } \\
\text { Ila }\end{array}$ & $\begin{array}{c}\text { Group } \\
\text { Ilb }\end{array}$ & $\begin{array}{l}\text { Group } \\
\text { Ilc }\end{array}$ & $\begin{array}{l}\text { Group } \\
\text { Ild }\end{array}$ & $\begin{array}{c}\text { Group } \\
\text { Ile }\end{array}$ & $\begin{array}{c}\text { Group } \\
\text { III }\end{array}$ & Unassigned \\
\hline Amborella trichopoda & 29 & 3 & 7 & 2 & 4 & 5 & 2 & 4 & 5 & 3 \\
\hline Arabidopsis thaliana & 72 & 2 & 14 & 3 & 8 & 18 & 7 & 8 & 14 & 2 \\
\hline Capsicum annuum & 66 & 4 & 16 & 4 & 6 & 13 & 11 & 7 & 9 & 4 \\
\hline Catharanthus roseus & 48 & 4 & 11 & 3 & 5 & 13 & 4 & 7 & 5 & 4 \\
\hline $\begin{array}{c}\text { Oryza sativa ssp. } \\
\text { japonica }\end{array}$ & 93 & 6 & 15 & 4 & 8 & 16 & 7 & 10 & 34 & 5 \\
\hline Rauvolfia serpentina & 49 & 5 & 10 & 2 & 4 & 12 & 5 & 5 & 5 & 11 \\
\hline Solanum lycopersicum & 78 & 3 & 15 & 5 & 8 & 17 & 6 & 17 & 11 & 2 \\
\hline Solanum tuberosum & 75 & 9 & 14 & 5 & 6 & 14 & 7 & 15 & 14 & 9 \\
\hline Urticularia gibba & 65 & 7 & 16 & 4 & 4 & 18 & 7 & 11 & 5 & 7 \\
\hline
\end{tabular}

WRKY TFs identified from the sequenced genomes of $A$. trichopoda, A. thaliana, C. annuum, O. sativa, S. lycopersicum, S. tuberosum, and U. gibba. C. roseus and R. serpentina WRKY TFs were identified from transcriptome sequences in the MPGR database. Complete and partial WRKY domain containing proteins were identified using the NCBI Conserved Domain Database. The presence of WRKY domains were manually verified and phylogenetic analyses were conducted to determine WRKY subgroups for each of the species.

\section{Phylogenetic analysis of Catharanthus WRKY TFs}

To determine the relationship among Catharanthus WRKY TFs, a phylogenetic tree was constructed with 282 WRKY domains from 243 TFs from Catharanthus, Amborella trichopoda, Arabidopsis and rice (Oryza sativa ssp. japonica) (Figure 1). WRKY sequences from Chlamydomonas reinhardtii (XP_001692342), Dictyostelium discoideum (XP_643786), and Giardia lamblia (XP_001708807) were included as an outgroup. Additional, outgroup sequences include human GCMa (BAA13651) and FLYWCH CRAa (EAW85450). We used 84 and 105 WRKY domains from 72 and 94 TFs, from Arabidopsis and rice respectively, to construct the phylogenetic tree. Thirty-five domains from 29 Amborella WRKY TFs were also included in the phylogenetic analysis [76]. We incorporated the WRKY sequences of Amborella, an evolutionary basal angiosperm, to reduce long-branch attractions during phylogenetic tree construction. Amborella was selected over Physcomitrella patens (moss) and Selaginella moellendorffii (spikemoss) since the WRKY sequences from this phylogenetically important species remains unreported yet provides valuable insights about WRKY evolution. The phylogenetic tree contained 58 domains from 48 CrWRKY TFs (Additional file 10: Table S6). To ascertain potential functions, we compared Catharanthus and Arabidopsis WRKY TFs by identifying orthologs. We identified 11 group I, 32 group II, and five group III WRKY TFs in Catharanthus. Group II WRKY TFs can be classified into groups IIa, IIb, IIc, IId, or IIe [32]. In Catharanthus, we identified three group IIa, five group IIb, thirteen group IIc, four group IId and seven group IIe WRKY TFs.

Evolutionarily, group I WRKY TFs, such as those found in algae, are some of the most ancient of WRKYs $[29,73]$. Recent evidence suggest that the group I WRKYs, and other WRKY TFs, originated from an ancestral group
IIc-like domain [31]. As previously reported for this group, ten group I CrWRKYs contained two WRKY domains with the N-terminal domain forming a separate clade and the C-terminal WRKY domains forming part of the group IIc clade [29,73]. To identify orthologs and paralogs in Amborella, Arabidopsis, Catharanthus and rice, we used OrthoMCL [77]. We found Catharanthus contains six coorthologs (CrWRKY2, CrWRKY3, CrWRKY4, CrWRKY5, CrWRKY8 and CrWRKY51) to AtWRKY33 (Table 4). According to the phylogenetic tree, CrWRKY5 is most closely related to AtWRKY33 (Figure 1).

Group IIa was the only group of WRKYs that had similar numbers between Catharanthus and Arabidopsis. Rice had four group IIa WRKY TFs whereas both Arabidopsis and Catharanthus each contained three. The three group IIa WRKYs from Catharanthus are coorthologs to AtWRKY40 (Table 4).

Previous reports indicate some plants contain variants of the highly conserved WRKYGQK domain, such as WRKYGKK, WRKYGEK, WRKYGSK, among others [29]. Variation in this region can reduce, eliminate, or alter DNA binding activity [78]. WRKY TFs with variants of the consensus sequence may recognize different cis-elements. We found AtWRKY50, AtWRKY51, and AtWRKY59 belong to the group IIc WRKY subfamily and possess a WRKYGKK motif as previously reported [32]. Two CrWRKYs were identified that contain variants of the highly conserved WRKYGQK motif. CrWRKY23 and CrWRKY32 contain WRKYGKK and WRKYGRK sequence motifs, respectively. Mutagenesis of the conserved glutamine was previously demonstrated to reduce, but not eliminate, DNA binding [78]. More recently, AtWRKY50 was found to generally bind the GAC core of the W-box with less preference for $5^{\prime}$ or $3^{\prime}$ bases [31]. Therefore, both WRKYGKK and WRKYGRK variants are expected to still bind DNA. Nictotiana tabacum WRKY12, containing a 


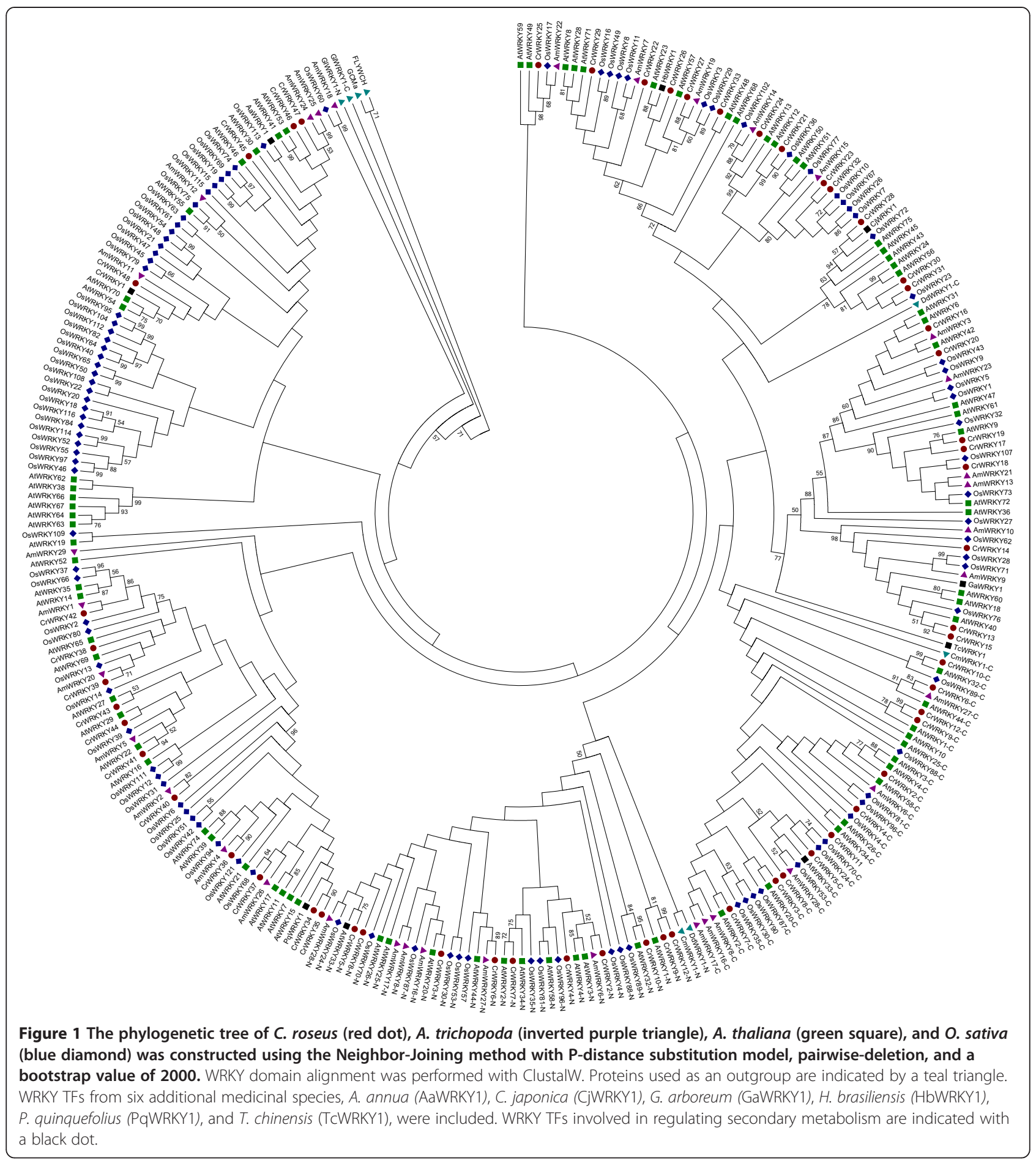

WRKYGKK motif, has been found to bind the WK-box ciselement (TTTTCCAC), but not the W-box, which regulates expression of the plant defense gene PATHOGENESIS RELATED1 [79]. However, Capsicum annuum WRKY1, a WRKYGKK motif WRKY TF involved in plant defense, can still recognize the W-box [80]. The phylogeny and protein alignment of the DNA-binding WRKY domains of
CrWRKY23, CaWRKY1 and NtWRKY12 revealed more similarity of CrWRKY23 to CaWRKY1 (Figure 2A and B), suggesting that despite the variant WRKYGKK motif, CrWRKY23 likely still recognizes the W-box element or at least the GAC core. Although Hordeum vulgare WRKY46 (SUSIBA2) contains a WRKYGQK motif, HvWRKY46 recognizes the Sugar Responsive (AATAGAAAA) and W-box 
Table 4 Orthologs and paralogs for Arabidopsis and Catharanthus WRKY found using OrthoMCL

\begin{tabular}{ll}
\hline Catharanthus roseus & Arabidopsis \\
\hline CrWRKY1, CrWRKY48, CrWRKY52 & AtWRKY54, \\
CrWRKY2, CrWRKY3, CrWRKY4, CrWRKY5, CrWRKY8, & AtWRKY20, \\
CrWRKY51 & \\
CrWRKY6 & AtWRKY44 \\
CrWRKY7 & AtWRKY2 \\
CrWRKY9, CrWRKY12 & AtWRKY1 \\
CrWRKY10 & AtWRKY32
\end{tabular}

CrWRKY13, CrWRKY14, CrWRKY15

CrWRKY16, CrWRKY20

CrWRKY17, CrWRKY18

CrWRKY19

CrWRKY21, CrWRKY22, CrWRKY26, CrWRKY27, CrWRKY29, CrWRKY33

CrWRKY23, CrWRKY32

CrWRKY24

CrWRKY25

CrWRKY28

CrWRKY30, CrWRKY31

CrWRKY34, CrWRKY35

CrWRKY36

CrWRKY37

\section{CrWRKY38}

CrWRKY39

CrWRKY41, CrWRKY43

CrWRKY42

CrWRKY45, CrWRKY46, CrWRKY47
AtWRKY40

GaWRKY1

AtWRKY6, AtWRKY31, AtWRKY42

TCWRKY1

AtWRKY72

AtWRKY9

AtWRKY8, AtWRKY12, AtWRKY23, AtWRKY28, AtWRKY48, AtWRKY51,

HbWRKY1 AtWRKY57, AtWRKY71

\section{AtWRKY50}

AtWRKY13

AtWRKY49

AtWRKY75

CjWRKY1

AtWRKY24, AtWRKY43, AtWRKY56

AtWRKY7

PqWRKY1

AtWRKY21

\section{AtWRKY11, AtWRKY17}

AtWRKY69

AtWRKY65

AtWRKY22, AtWRKY27

AtWRKY14, AtWRKY35

AtWRKY41, AtWRKY46, AtWRKY53

AaWRKY1

WRKYs in 'bold font' are jasmonate responsive, either according to the literature or by our findings in this study. WRKY highlighted in italics are TFs known to regulate secondary metabolism in A. thaliana, Artemisia annua, C. rosues, Coptis japonica, Gossypium arboreum, Hevea brasiliensis, Panax quinquefolius, and Taxus chinensis.

\section{A}

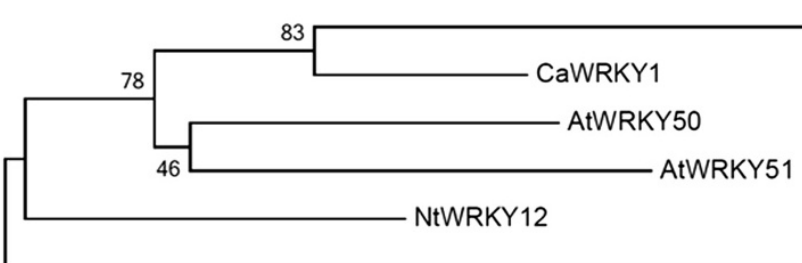

CrWRKY23

AtWRKY59

\section{B}

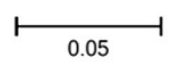

Figure 2 The phylogenetic relationship and alignment of CrWRKY23 to other WRKYGKK containing WRKY transcription factors. A. The phylogenetic tree was constructed in MEGA5 using the Neighbor-Joining method with P-distance substitution model, pairwise-deletion, and a bootstrap value of 2000. The tree is unrooted and branch lengths drawn to scale to evolutionary distances. Alignment was performed using ClustalW. B. Alignment of WRKY domain sequence was performed using ClustalW. 
cis-elements to regulate barley genes involved in starch metabolism [81]. This leaves open the possibility that some Catharanthus WRKY TFs may not recognize W-box ciselements.

Group III WRKY TFs are believed to have dramatically expanded during the evolution of angiosperms and can be classified into different subgroups depending on the species [34,72]. Arabidopsis contains fourteen group III WRKY TFs which are further divided into eight group IIIa and six group IIIb. In Arabidopsis, most group III WRKY transcription are induced by plant pathogens [34]. We identified only five group III WRKY TFs in Catharanthus. Similarly, we identified 5 group III WRKYs in serpentwood and bladderwort (Table 3). Proportionally, the number of group III CrWRKY TFs is the smallest compared to Arabidopsis. The low number of group III CrWRKYs, and similar number from serpentwood and bladderwort, suggests this group has not undergone significant expansion such as occurred in rice or Arabidopsis [72]. CrWRKY1 and CrWRKY48 were found to be coorthologs to AtWRKY70 and AtWRKY54 (Table 4). AtWRKY70 modulates SA and jasmonate signaling [36]. Interestingly, CrWRKY1 differentially directs the flow of unknown precursors into TIA products [46], a feature possibly governed by its jasmonate responsive gene expression. CrWRKY45, CrWRKY46, and CrWRKY47 are coortholgs of AtWRKY41, AtWRKY46, and AtWRKY53. AtWRKY46 and AtWRKY53 are partially functionally redundant in regulating plant defense [43].

We previously reported the role of CrWRKY1 in regulating gene expression and TIA accumulation in Catharanthus [46]. CrWRKY1 is a group III WRKY with overall protein sequence homology closest to AtWRKY70, and corresponds to MPGR contig number Cra16284. Phylogenetically, CrWRKY1 is located towards the base of the group III clade and does not clearly group with its Arabidopsis or rice orthologs (Additional file 11: Figure S5A). To identify the unique feature of CrWRKY1, we analyzed the protein sequence alignment. The invariant tryptophan starting the WRKYGQK motif was used as the reference point for comparing alignments. Alignment of CrWRKY1 to other group III WRKY TFs revealed that CrWRKY1 lacks an amino acid between the two conserved cysteine residues at positions 21 and 29 (Additional file 11: Figure S5B). The closest rice WRKY TFs, OsWRKY21, OsWRKY61 and OsWRKY47, all have altered spacing within the WRKY domain sequence. OsWRKY47 possesses an additional proline residue between the WRKYGQK sequence and the conserved arginine residue at position 16 [32]. The conserved arginine at position 16 was changed to threonine followed by a TQS motif in OsWRKY61. OsWRKY47 contains an extra DDP sequence between positions 41 and 42 compared to all other Arabidopsis, rice, and Catharanthus group III WRKY TFs. The altered spacing in the WRKY domain may give these WRKY TFs unique structural properties important for target gene regulation.

\section{Expression profiling reveals multiple Jasmonate responsive CrWRKYs}

MPGR provides RNA-sequencing based expression data from different tissues for all sequenced medicinal plants. For Catharanthus, RNA-sequencing data is also available for different tissues, seedlings, cell suspension cultures, and hairy root cultures. These data provide an opportunity to understand the induction of WRKY genes in response to conditions that induce the TIA pathway. Furthermore, several treatments allow for comparison of induction to the same hormone in varying tissues. In response to MeJA, a potent and important elicitor of natural product formation, including TIAs, in Catharanthus and other medicinal species [82-85], MPGR expression data indicates multiple WRKY TFs are either up or down regulated in Catharanthus.

To identify or validate WRKY TFs that are up- or down regulated by MeJA, we performed qRT-PCR on whole plant samples (root, stem, and leaves) that were collected from one month old soil grown plants at 0, 1, 2, and 4 hours after MeJA treatment. Successful induction with MeJA was verified by measuring JAZ2 expression (Figure 3A). To determine which WRKY TFs were possible regulators of TIA production, we sought to measure the expression of multiple pathway genes such as $G 10 H, T D C$, and STR (Figure 3B). These genes were selected to represent early (G1OH and TDC) and middle portions (STR) of the TIA pathway. Any WRKY induced prior or simultaneously to these genes possibly could regulate that corresponding portion of the pathway and any subsequent segments.

We selected at least two genes from each CrWRKY subgroup. Four genes (CrWRKY5, CrWRKY8, CrWRKY13, and $C r W R K Y 28$ ) were selected based on involvement of their orthologs in regulating secondary metabolism genes in other species. Analysis of sixteen CrWRKYs identified twelve which displayed significant changes in expression in response to jasmonate (Figure 3C-D). The fold change for most CrWRKYs with a significant response to MeJA was 2 fold or less (Figure 3C-D), similar to our microarray findings for jasmonate responsive Arabidopsis WRKYs (Additional file 3: Table S3). CrWRKY8 was up-regulated 1 hour after MeJA treatment then decreased by 4 hours after treatment. CrWRKY5 was down-regulated significantly at both 2 and 4 hour after MeJA treatment. CrWRKY13, similar to the ABA responsive AtWRKY40, was significantly up-regulated 1 and 2 hours after MeJA treatment. CrWRKY38 was up-regulated by 2 hours after MeJA treatment. CrWRKY18, CrWRKY21, CrWRKY41, CrWRKY45, and CrWRKY48 were all significantly downregulated at all time points after MeJA treatment. Two 


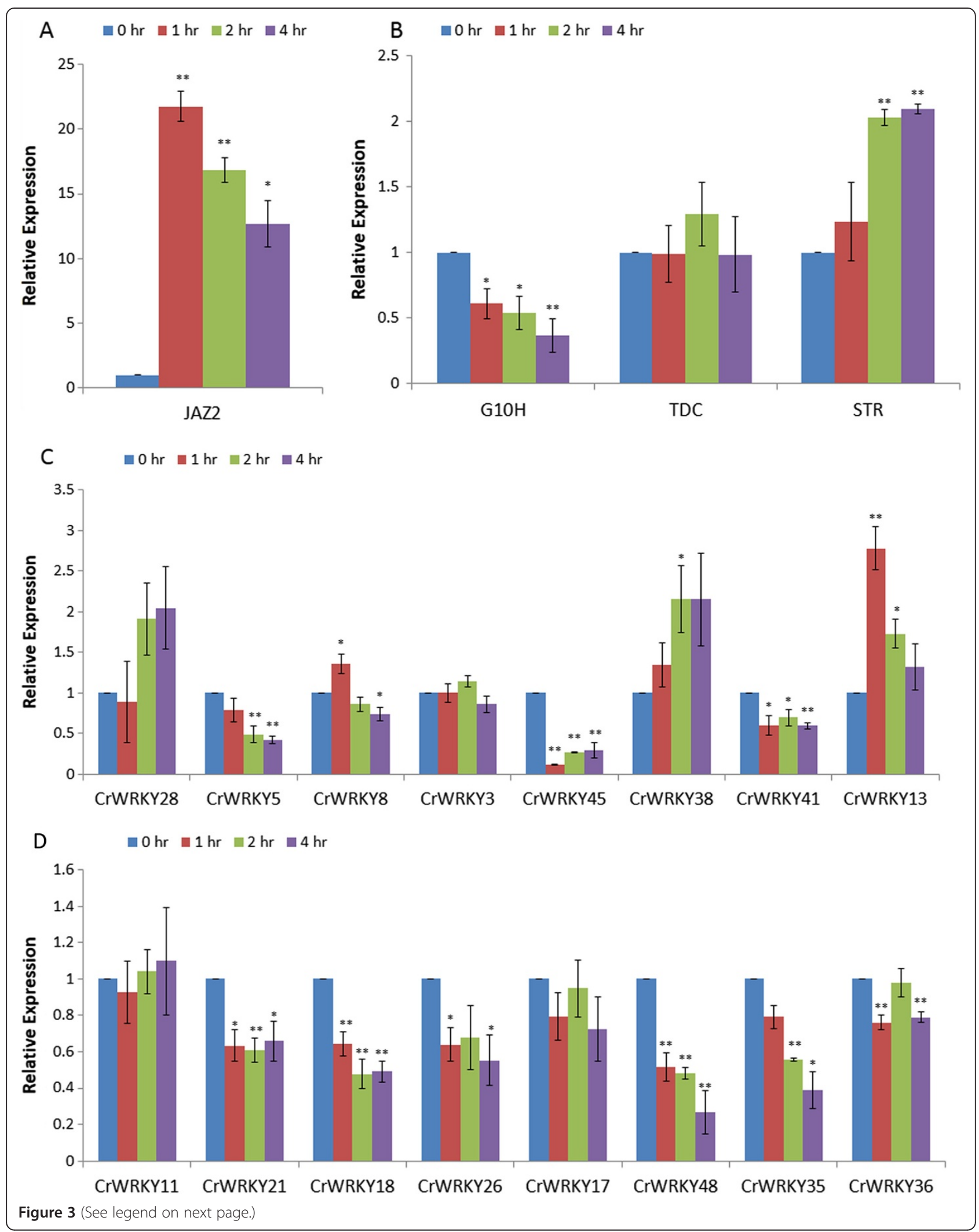


(See figure on previous page.)

Figure 3 Quantitative reverse-transcription PCR (qRT-PCR) for quantification of gene expression was performed on mature Catharanthus plants. Three whole plants were combined for each biological replicate. Each time point consisted of three biological replicates. Three technical replicates were measured per time point sample. A. Expression of Catharanthus JAZ2 transcripts were determined in after 0, 1, 2, or 4 hours of MeJA treatment. B. Expression of the TIA biosynthetic genes G1OH, TDC, and STR. C-D. Expression of 16 Catharanthus WRKY transcription factors in response to $0,1,2$, and 4 hours of MeJA treatment. Significant and highly significant, $p$-value $<0.05$ or 0.01 respectively, changes in gene expression were determined using a Student's T-test.

WRKYs, CrWRKY26 and CrWRKY36, had a bimodal expression pattern that was down-regulated at 1 and 4 hours, but not 2 hours, after MeJA treatment. A bimodal expression pattern has been observed for some regulators of the TIA pathway [15]. CrWRKY35 was down-regulated 2 and 4 hours after treatment. Nine of 12 CrWRKYs analyzed were down-regulated to MeJA treatment. In total at least 25\% (12 of 48), and probably more, of CrWRKY TFs are regulated by jasmonate. Of the twelve jasmonate responsive CrWRKYs, nine have an AtWRKYs ortholog which were either previously reported and/or identified here by microarray analysis ( $\mathrm{p}<0.05$ and survived B-H FDR) as differentially regulated by jasmonate (Figure $3 \mathrm{C}-\mathrm{D}$, Additional file 2: Table S2). When compared to the less stringent list of AtWRKYs which had expression significantly changed $(\mathrm{p}<0.05)$ in response to jasmonate, but did not survive the B-H FDR, all twelve CrWRKYs have orthologs to jasmonate responsive AtWRKYs.

To identify potential WRKYs regulating TIA biosynthesis through the jasmonate signaling pathway, we compared the induction times of WRKY genes (Figure 3C-D) to early and mid- biosynthetic genes of the pathway (Figure 3B). Similar to previous reports in cell cultures [15], induction of STR by MeJA began approximately 2 hours after treatment. However, expression of $\mathrm{G} 10 \mathrm{H}$ decreased starting at 1 hour after MeJA treatment, and was further downregulated 4 hours after treatment (Figure 3B). TDC transcript levels remained unchanged to MeJA treatment in mature Catharanthus plants. Prior reports of TDC [7,13,86-88] and $G 10 H$ [13,86-88] transcript induction by jasmonate treatment was identified in seedlings, hairy roots, or cell cultures; however, our experiments were performed in intact mature Catharanthus plants. Expressions of WRKY TFs possibly contributing to TIA regulation are predicted to be altered before early and mid steps of the TIA pathway. Expression of CrWRKY8, CrWRKY13, CrWRKY18, CrWRKY21, CrWRKY26, CrWRKY36, CrWRKY41, CrWRKY45 and CrWRKY48 changed by 1 hour after MeJA treatment indicating these WRKYs could possibly regulate the expression of early TIA pathway genes. Altered expression of all twelve CrWRKYs responding to MeJA occurred by 2 hours after treatment, the same time at which significant induction of STR occurred. Contrary to TDC and $\mathrm{G} 10 \mathrm{H}$, which contain four and one W-boxes in their promoters respectively [46,89], the characterized STR promoter does not contain any
W-box elements for WRKYs to bind, but this does not exclude the possibility that WRKY regulate other TFs controlling STR expression. The spatio-temporal regulation of CrWRKYs, by reducing TDC responsiveness and down-regulating $G 10 H$, is one possible reason why mature Catharanthus plants do not accumulate TIA in response to jasmonate treatment [90]. These findings suggest that all CrWRKYs we ascertained as differentially expressed in response to jasmonate are possible regulators of early and middle steps of TIA biosynthesis. Presumably, these CrWRKY could also regulate downstream steps of the pathways which are temporally expressed later.

\section{The Jasmonate Response of Catharanthus WRKY Varies Among Plant Culture Conditions}

We sought to determine the similarities between our qRT-PCR results and the transcriptome data published by MPGR. As we used one month-old plants to quantify gene expression, and no data on MeJA treated mature plants are provided by MPGR, we correlated our data to three different datasets each representing one aspect of our samples ( 5 day MeJA treated seedlings, 6 hour MeJA treated cell suspension cultures, and 24 hour MeJA treated hairy root cultures). Seedlings treated with MeJA most closely represent our samples in physiology as both are whole plant tissues; however, the MPGR dataset used seedlings rather than mature plants, which may respond to MeJA differently [90]. While cell cultures are considerably different in physiology from whole plants, the earliest time sample (6 hours after MeJA treatment) was closest to our sample times of 1,2 , and 4 hours after MeJA treatment. Hairy root cultures require several weeks to develop to sufficient size; therefore, the age of this tissue most likely represents a similar age as our plant samples, despite our shorter MeJA treatment time. The Pearson correlation coefficient was calculated to measure the relationship between the datasets. CrWRKY11 and CrWRKY21 were excluded from the correlations as they appear two times in the MPGR datasets without expression values. The correlation between MPGR seedling and cell culture datasets, as well as between cell culture and hairy root datasets, was quite low $(r=0.179$ and $r=0.227$ respectively), indicating considerable difference in CrWRKY response to MeJA in cell culture systems. However, there was a high 
correlation between seedling and hairy root MPGR datasets $(r=0.892)$ for MeJA treated CrWRKY genes. Our qRT-PCR data showed that the correlations ranged from 0.061 , between 1 hour MeJA treated plants and 24 hour MeJA treated hairy roots, to 0.790 , between 1 hour MeJA treated plants and 6 hour MeJA treated cell suspension cultures (Figure 4A). Overall, the three MPGR datasets correlated well with all three time points of qRT-PCR data (median value of the 9 correlations = 0.555 ) indicating similar expression changes in response to jasmonate treatment. Increasing time after MeJA treatment in whole plants increased the correlation with seedling and hairy root cultures. Cell cultures, despite higher similarities in the 4 hour MeJA treated plant and 6 hour MeJA treated cell culture time frame, showed a lower correlation between the 1 hour MeJA treated plant and 6 hour MeJA treated cell cultures. Similar to Arabidopsis, these findings in Catharanthus suggest significant differences exist between jasmonate response in various cultural conditions, including intact seedlings, adult plants, cell cultures, and hairy root cultures.

Gene expression clusters often contain genes with related functions [58], including those in natural product formation [91]. Recently, clustering of MPGR expression data has aided the identification of Catharanthus IRIDOID SYNTHASE [92]. To identify potential clusters of CrWRKY TFs with similar expression pattern which may indicate WRKY functions, we performed a hierarchical clustering. Unsupervised agglomerative hierarchical clustering of 23 transcriptome gene expression datasets from MPGR revealed three primary clusters: a plant tissue cluster, a hairy root cluster, and a protoplast cluster (Figure 4B). Clusters of plant culture type indicate a greater difference between cultural conditions than between MeJA treatment. However, clear differences exist between MeJA treated and untreated samples within subgroups of each primary cluster. Clustering of CrWRKY gene expression revealed three primary clusters. CrWRKYs of cluster one were most up-regulated in different plant tissues, suggesting a role in plant development. The second cluster of WRKY genes is up-regulated in hairy root cultures. Members of cluster two may be important for regulating metabolism and resource direction into primarily root produced alkaloids, such as ajmalicine and serpentine. Identification of $C r W R K Y 1$, which plays a role in serpentine production, in cluster two supports this idea. CrWRKY46, ortholog to AaWRKY1, a trichome expressed WRKY in Artemisia annua, was also found in this cluster. CrWRKY34 and CrWRKY35 orthologous to PqWRKY1 which is suggested to regulate terpene biosynthesis in Panax quinquefolius (American ginseng) roots also occur in this cluster. The third cluster consisted of CrWRKY that were up-regulated in response to MeJA or yeast extract (YE), an elicitor of TIA biosynthesis, in protoplasts. Most
A

\begin{tabular}{|l|ccc|}
\hline & Seedling 5 day MeJA & Cell Culture 6 hr. MeJA & Hairy Root 24 hr. MeJA \\
\hline Plant 1 hr. MeJA & 0.103 & 0.790 & 0.061 \\
Plant 2 hr. MeJA & 0.615 & 0.413 & 0.555 \\
Plant 4 hr. MeJA & 0.690 & 0.307 & 0.660 \\
\hline
\end{tabular}

B

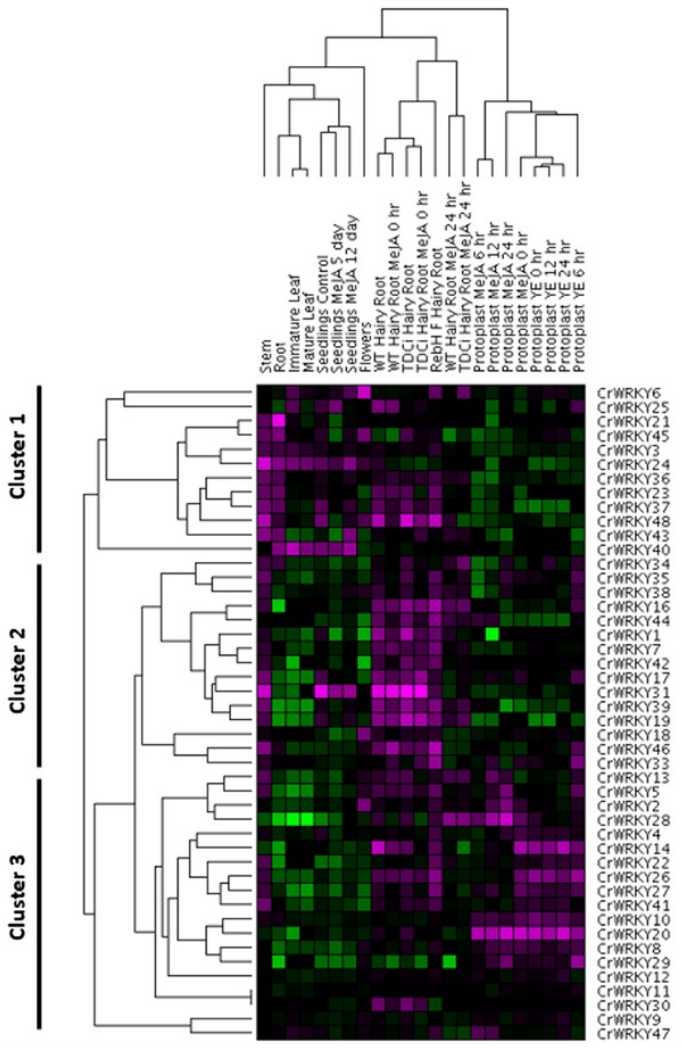

Figure 4 Pearson correlation analysis of WRKY expression data and hierarchical clustering of MPGR transcriptome data for the CrWRKY TF family. A. Pearson correlation between fold change of qRT-PCR expression and MPGR datasets for MeJA treated samples. Fold change for both sets was calculated using the reference gene EF1a as an internal control. B. Hierarchical cluster analysis of MPGR transcriptome data for the CrWRKY TF family was performed using GenePattern. The clustering method was a pairwise average linkage with distance measured using the Pearson correlation coefficient. Data was log transformed. The median value was subtracted from each row. Color is based on global expression with purple being up-regulated and green down-regulated.

of this cluster was also up-regulated in hairy root cultures. Most CrWRKYs orthologous to WRKYs regulating natural product formation in other species were identified in this cluster. Importantly, four CrWRKYs (CrWRKY2, CrWRKY5, CrWRKY13, and CrWRKY28), similar to those with known roles in secondary metabolism (Table 4), were identified as part of the same sub-cluster in cluster three. The four members of this cluster may play key roles in regulation of natural product formation in Catharanthus. A second subcluster of cluster three, composed of six members, contained five CrWRKYs (CrWRKY4, CrWRKY14, CrWRKY22, CrWRKY26, and CrWRKY27) which are orthologs to 
WRKY TFs regulating natural products in other species. The sixth member of this sub-cluster, CrWRKY41, is also a jasmonate responsive WRKY. Members of this subcluster may also be important for regulation of natural products in Catharanthus. CrWRKY TFs, determined by qRT-PCR to be MeJA responsive, were distributed across all three clusters, indicating jasmonate broadly regulates WRKYs from each cluster.

\section{Predicted role of CrWRKY orthologs in secondary metabolism}

Catharanthus produces alkaloids, terpenes and latex, all classes of compounds that contain biosynthetic genes involved in their production which have been implicated to be regulated by WRKY TFs in other species $[48,49,51]$. To associate biosynthesis of natural compounds with jasmonate-responsive CrWRKYs, we compared Catharanthus WRKY TFs to those known to control secondary metabolism in other plant species (Table 4). CjWRKY1 is involved in the regulation of the benzylisoquinoline alkaloid berberine [48]. In Catharanthus, CrWRKY28 grouped closely with CjWRKY1 (Figure 1). The ortholog of CjWRKY1 in Arabidopsis is AtWRKY75.

AtWRKY33 plays a role in regulating biosynthesis of camalexin, an indole ring and $\mathrm{N}$-containing defense molecule, and functions downstream of MITOGENACTIVATE PROTEIN KINASE 3 and 6 [52]. Recently, CrMPK3 was shown to regulate TIA accumulation [93]. As Catharanthus produces over 130 different TIA metabolites, the multiple coorthologs to AtWRKY33 may be important for regulating diverse products of this pathway. However, further experiments are needed to demonstrate whether the orthologous TFs in Catharanthus act downstream of CrMPK3 and are involved in TIA biosynthesis. Interestingly, CmWRKY1, from Chlamydomonas, was also an ortholog to AtWRKY33, suggesting a possible early function of this TF in defense and regulating secondary metabolism.

AaWRKY1, which is involved in regulating the accumulation of artemisinin, has three coortholgs in Catharanthus, CrWRKY45, CrWRKY46, and CrWRKY47 (Table 4). In Arabidopsis three group IIIa WRKYs, AtWKRY41, AtWRKY46, and AtWRKY53 are coortholgs to AaWRKY1. In Catharanthus, increased production of HMGR and terpenes have negatives effect on the accumulation of certain TIAs [94]. In A. annua, AaWRKY1 affects the expression of 3-HYDROXY-3-METHYLGLUTARYLCOA REDUCTASE (HMGR) [50], a rate limiting enzyme in the mevalonate pathway. Both AaWRKY1 and CrWRKY46 are jasmonate responsive genes; therefore, at least CrWRKY46 may have an evolutionarily conserved function in regulating the flux of carbon into Catharanthus terpenes.
The rate limiting enzyme in the production of paclitaxel, DBAT, is regulated by TcWRKY1 [47]. Phylogenetically, TcWRKY1 is basal to the group IIa and IIb clades (Figure 1). Catharanthus CrWRKY16 and CrWRKY20 were identified as coorthologs to TcWRKY1 (Table 4). In Arabidopsis, the group IIb AtWRKY6, AtWRKY31, and AtWRKY42 were found to be coortholgs to TcWRKY1 (Table 4).

GaWRKY1, from cotton, regulates a sesquiterpene cyclase leading to the production of gossypol [49]. AtWRKY40 was found to be the Arabidopsis ortholog to GaWRKY1 (Table 4). AtWRKY18 and AtWRKY60 formed their own ortholog group independent of AtWRKY40. Catharanthus, however, contains three coortholgs to GaWRKY1, CrWRKY13, CrWRKY14, and CrWRKY15. As with AtWRKY40, these CrWRKYs may have a role in negative regulation of ABA response [95] and positive regulation of jasmonate responses [38]. Supporting this idea, like AtWRKY40 and GaWRKY1, we found expression of CrWRKY13 was induced by jasmonate treatment. Drought, salinity, and cold all affect TIA accumulation in Catharanthus [12,96], thus at least CrWRKY13 may function in regulating the accumulation of TIAs in response to abiotic stress and plant defense.

$H b W K R Y 1$ expression is related to latex production in rubber trees [51]. In Catharanthus, CrWRKY21, CrWRKY22, CrWRKY26, CrWRKY27, CrWRKY29, and CrWRKY33 are coorthologs to HbWRKY1. Six coothrologs (AtWRKY8, AtWRKY12, AtWRKY23, AtWRKY28, AtWRKY48, AtWRKY51, and AtWRKY71) to HbWRKY1 exist in Arabidopsis (Table 4). Like HbWRKY1, at least four Arabidopsis (AtWRKY8, AtWRKY28, AtWRKY48, and AtWRKY51) and two Catharanthus WRKYs (CrWRKY21 and CrWRKY26) are regulated by jasmonate. Phylogenetically, the group IIc AtWRKY23 and CrWRKY26 are the WRKYs most similar to HbWRKY1 in Arabidopsis and Catharanthus, respectively (Figure 1). As Catharanthus also produces a latex compound, the jasmonate regulated CrWRKY26 or one of the other paralogs, may function in the regulation of latex or terpene production in Catharanthus.

Heterologous over-expression, in Arabidopsis, of the MeJA responsive American ginseng WRKY TF, PqWRKY1, increased drought and salt stress tolerance, in addition to regulating terpene biosynthetic genes [53]. AtWRKY7 in Arabidopsis and CrWRKY34 and CrWRKY35 in Catharanthus are orthologs to PqWRKY1 (Table 4). Contrary to the report by Sun et al. [53], which classifies PqWRKY1 as a group IIc WRKY, we found PqWRKY1 actually falls within the IId subgroup when compared to the entire Arabidopsis WRKY family (Figure 1). AtWRKY7, CrWRKY35, and PqWRKY1 are each regulated by jasmonate supporting the possible conserved evolutionary function of these proteins in regulating terpene biosynthesis. 
Kalde et al. [34] reported a role of most Arabidopsis group III WRKY TFs in plant defense. Overall, no clear trend was observed for WRKY TFs possibly involved in secondary metabolism belonging to a specific group or subgroup. Further work is needed to verify the predicted roles of these CrWRKYs in the regulation of secondary metabolism.

Comparative genetics across species has provided invaluable information that lead to the isolation and functional understanding of several key regulators in natural product formation. In Arabidopsis, the bHLH factor AtMYC2 is known as a central regulator of jasmonate signaling pathway. The orthologs of $A t M Y C 2, C r M Y C 2$ and NtMYC2, from Catharanthus and tobacco, respectively, have thus been isolated and characterized. While Arabidopsis does not produce TIAs or nicotine, CrMYC2 and NtMYC2 act in the jasmonte signaling pathway to regulate biosynthesis of these metabolites $[16,97]$. Moreover, AtMYC2 can bind the jasmonate-responsive elements present in the promoter of Catharanthus ORCA3, an AP2/EFR TF gene, and activates its expression, illustrating the conserved nature of these orthologous regulators [98]. These reports further strengthen our reasoning for cross-species comparison of WRKY TFs from Catharanthus, Arabidopsis, and other medicinal plant to identify regulators conserved in jasmonate response and possibly secondary metabolite production.

Comparison of CrWRKYs with orthologs from other species, that are known to regulate natural products or respond to jasmonate treatment, helped us develop a model for WRKY regulation of TIA biosynthesis in Catharanthus (Figure 5). In this model, jasmonate acts as a central regulator of the TIA pathway with both positive and negative effects on WRKYs. Phytohormones, including ABA, ethylene and gibberellin (GA), are also likely involved in CrWRKYs regulation. Overall, this work provides a fundamental base for which future experiments can be designed to help elucidate the molecular mechanisms controlling the biosynthesis of highly valuable TIAs.

\section{Conclusion}

Taken together, our results illustrate a role for the Arabidopsis WRKY family in regulating jasmonate response. These findings strengthened our reasoning for investigating Catharanthus jasmonate responsive WRKY TF which are potentially involved in regulation of TIA biosynthesis. Results from Arabidopsis and Catharanthus suggest that the regulation of WRKY gene expression in response to jasmonate is dependent upon environmental and spatio-temporal context. Such information can be important in designing metabolic engineering projects. Furthermore, we identified numerous jasmonate responsive orthologs between AtWRKY and CrWRKY TFs that may be functionally conserved or partially conserved.

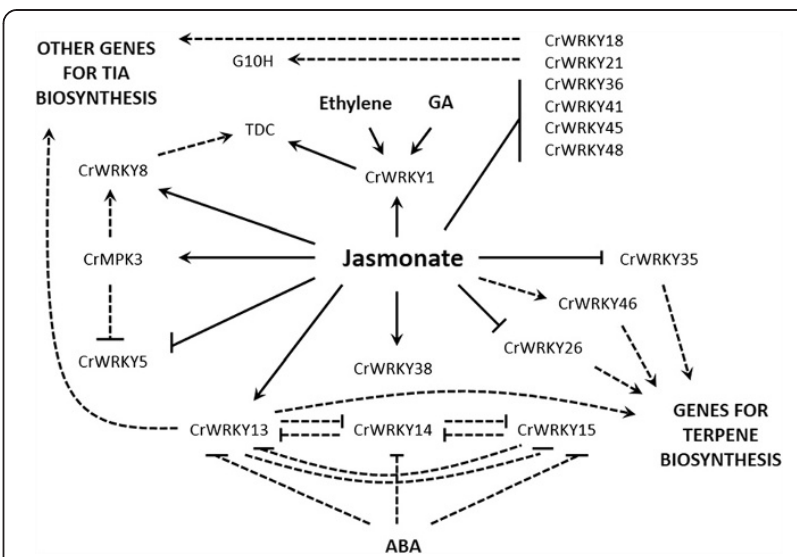

Figure $5 \mathrm{~A}$ model of WRKY TFs function in Catharanthus based on expression data and known roles in A. thaliana. The model depicts CrWRKYs which were either similar to a WRKY with a known role in regulating natural product formation in another species or had transcript level differentially expressed in response to MeJA treatment. Jasmonate has both positive and negative effects on CrWRKY transcript accumulation which is possibly important for fine-tuning TIA and terpene biosynthetic gene expression. The hormones abscisic acid (ABA), ethylene, and gibberellin (GA) also are likely important for regulation of Catharanthus WRKYs. Solid lines depict known regulations and dashed lines indicate hypothetical regulatory interactions.

The jasmonate responsive CrWRKYs are potential candidate TFs for having key roles in modulating jasmonate signaling and regulating TIA biosynthesis. Information on how AtWRKYs response to various phytohormones and stresses may also apply to Catharanthus. This information may be useful for understanding how other phytohormones also contribute to the regulation of TIA production. Moreover, elucidation of CrWRKY functions may provide valuable insights into the regulation of natural product biosynthesis in other medicinal plants.

\section{Methods}

\section{Plant growth conditions}

Catharanthus 'Little Bright Eyes' seeds were surface sterilized and were germinated in the dark at $30^{\circ} \mathrm{C}$ for 3 days on MS plates, before being then transferred to an ambient temperature $24 \mathrm{~h}$ light photoperiod tissue culture room for an additional 4 days. Seedlings were transferred to soil and grown at ambient temperature under $24 \mathrm{~h}$ light. Samples were collected from 1 month-old Catharanthus plants treated with MeJA for $0,1,2$, or 4 hours. The MeJA experiment was performed once with each time having three replicates. Three plants were combined from each replicate time sample. MeJA treatment consisted of spray application of $100 \mu \mathrm{M}$ MeJA then placing plants under a clear plastic dome sealed with tape. Whole plants were harvested, roots quickly washed, and then frozen in liquid nitrogen. 


\section{WRKY TF Identification}

Contigs translated into protein sequence were downloaded from the MPGR. The single longest copy of each contig translated into protein sequence was identified using Microsoft Excel. Each unique contig number, which translated into some protein sequence, was determined to represent a unique gene distinguished by locus number. To differentiate potential WRKY genes each distinct contig locus number, but not different length variants of the same locus number, were considered as a unique product. As observed in Arabidopsis and other species, the multiple contig copies that comprise many of the loci may represent splice variants, not fully sequenced transcripts or different alleles. A Microsoft Excel file containing all protein encoding contigs was searched to manually identify WRKY and WRKYGQK invariant motif containing proteins. A FASTA file of the single longest protein encoding contig for the entire genome was submitted to the NCBI CDD and PlantTFcat servers to identify whole and partial WRKY domains containing contigs. The process was performed for Amborella trichopoda, Arabidopsis thaliana, Capsicum annuum, Catharanthus roseus, Oryza sativa ssp. japonica, Rauvolfia serpentina, Solanum lycopersicum, Solanum tuberosum, and Urticularia gibba. A file containing WRKY TFs from Catharanthus, Amborella, Arabidopsis, and rice was submitted to OrthoMCL [77] to identify orthologs and paralogs. WRKY TFs involved in regulating secondary metabolism from other species were also included. GenBank accession numbers for medicinal plant WRKY TFs included are: AaWRKY1 (FJ390842), CjWRKY1 (AB267401), TcWRKY1 (JQ250831), GaWRKY1 (AY507929), HbWRKY1 (GU372969), and PqWRKY1 (AEQ29014).

\section{Phylogenetic tree construction}

The unrooted phylogenetic trees for Catharanthus, Amborella, Arabidopsis, and Oryza sativa ssp. japonica and medicinal plant WRKY TFs were constructed using the MEGA5 software. The neighborhood joining method, with bootstrap values of 2000, was utilized to conduct the phylogeny test. The analysis used p-distance of amino acid sequence to determine substitution rate. Gaps or missing data were excluded as needed, according to the pairwise deletion option. Phylogenetic trees analyzing the bladderwort, pepper, potato, serpentwood, and tomato WRKY families were constructed in the same way.

\section{RNA extraction}

RNA was extracted using an extraction buffer composed of 1\% 1,5-naphthalenedisulfonic acid and 4\% p-aminosalicylic acid prepared in diethylpryocarbonate (DEPC) treated water. A $5 \mathrm{M}$ sodium hydroxide solution was added until the extraction buffer was fully dissolved. For each RNA sample $5 \mathrm{~mL}$ of extraction buffer solution was mixed with $5 \mathrm{~mL}$ of liquefied phenol. Ground samples were added to the extraction buffer/phenol solution, vortexed 1 minute, $5 \mathrm{~mL}$ of chloroform added, then vortexed again. Samples were spun down for 10 minutes at $6000 \mathrm{rpm}$ at $4^{\circ} \mathrm{C}$. The aqueous phase was transferred to a $50 \mathrm{~mL}$ centrifuge tube and $1 / 10^{\text {th }}$ the volume of $3 \mathrm{M}$ sodium acetate $(\mathrm{pH}$ 5.3) was added along with 2 times the volume of chilled $100 \%$ ethanol. Samples were incubated on ice $1 \mathrm{hr}$ prior to centrifugation. The supernatant was discarded and the pellet dried for 30 minutes. The dried pellet was resuspended in $4 \mathrm{~mL}$ of autoclaved DEPC treated water and $2.5 \mathrm{~mL}$ of $8 \mathrm{M}$ lithium chloride and incubated at $4^{\circ} \mathrm{C}$ overnight. The RNA was then precipitated by centrifuging at the above. The pellet was rinsed with chilled DEPC treated 80\% ethanol. The ethanol was decanted and the RNA allowed to dry for 30 minutes before resuspending in sterile DEPC treated water.

\section{cDNA Synthesis and gene expression}

Synthesis of first strand cDNA from total RNA isolated from plant tissue and quantitative reverse transcription polymerase chain reaction (qRT-PCR) were performed as previously reported [89]. Samples for the MeJA treatment consisted of 3 biological replicates each with 3 technical replicates. The comparative cycle threshold method was used to measure the transcript levels. All primers used for qRT-PCR can be found in Additional file 12: Table S7. Significant differences in gene expression were calculated using the Student's T-test. P-values of 0.05 and 0.01 were considered significant and highly significant, respectively.

\section{$5^{\prime}$ and $3^{\prime}$ rapid amplification of CDNA Ends (RACE) and cloning}

5' and 3' RACE was performed using the RACE kit (Invitrogen) as directed by the manufacturer. A nested set of PCRs was performed to isolate the target sequence. The first PCR reaction used the AAP primer and a gene specific primer; whereas the second nested reaction used the AUAP primer and a second gene specific primer. 3' RACE was performed as for gene expression CDNA synthesis with the modification of using the 3' AP primer to create a 3' adapter. The 3' target sequence was amplified through PCR using a nested set of gene specific primers and the adapter specific 3 ' AUAP primer. All 3' and 5' RACE primers are listed in Additional file 13: Table S8.

\section{Cloning and sequencing of partial WRKY domains}

Partial WRKY domain sequences to be cloned were amplified using $5^{\prime}$ or $3^{\prime}$ RACE. The sequence was then separated on an agarose gel and the DNA purified using a Wizard ${ }^{\circ}$ SV Gel and PCR Clean-Up System (Promega). The purified DNA was ligated into pGEM-T Easy vector 
(Promega). Plasmid isolation was performed using a Wizard $^{\circledR}$ Plus SV Minipreps DNA Purification System (Promega). 250-300 ng plasmid DNA were sequenced with either the T7 or SP6 primer using DTCS Quick Start (Beckmann) according to the manufacturer's protocol. Sequencing was performed with a $\mathrm{CEQ}^{\mathrm{rm}} 8000$ Genetic Analyzer System (Beckman Coulter) Sanger sequencer.

\section{Arabidopsis microarray analysis and gene expression}

Jasmonate treated microarray datasets were collected from NCBI, EMBL, and TAIR. RMA Express was used for array normalization of each experiment [99]. Background adjustment, quantile normalization, and median polish were applied. Data was exported as log transformed data then analyzed by two-way ANOVA using the MEV software [100]. Two-way ANOVAs were performed on each dataset to determine response to jasmonate treatment and another variable (genotype or time). Controls and probes not linked to a gene were eliminated post-ANOVA prior to application of the false discovery rate. The B-H FDR was calculated in Microsoft Excel according to Thissen et al. [95]. Significant differences $(p<0.05)$ were determined before and after application of the BenjaminiHochberg false discovery rate (B-H FDR). Significant differences before the B-H FDR was applied were both included because qRT-PCR for CrWRKY TFs indicated small (less than 2 fold), yet significant, changes to jasmonate treatment.

\section{Hierarchical clustering and correlations}

The Pearson correlation coefficient was calculated to measure the relationship between qRT-PCR and MPGR datasets. First, the fold change for Catharanthus WRKY genes from the MPGR dataset was calculated in reference to 0 hour control treatments. Differences between control and MeJA treated datasets were then adjusted using the Catharanthus reference gene EF1 $\alpha$ (Cra3894) as an internal control [13], as this gene was used as the internal control for qRT-PCR expression measurements. The correlation coefficient between fold changes in expression was calculated using Microsoft Excel.

Unsupervised agglomerative hierarchical clustering was performed using the GenePattern [96] website (http://genepattern.broadinstitute.org). The Pearson correlation was used as a distance measure for both row and column clustering. The clustering method was pairwise-average linkage with a row centering. Row centering was performed by subtracting the median value of each row. A global color scheme using a color gradient was applied for visualization. Purple, black, and green indicate increased gene expression, no change and decreased gene expression respectively. For Arabidopsis microarray data expression values were first analyzed with RMA Express and log transformed values exported for analysis with GenePattern $[93,96]$.

\section{Availability of supporting data}

Catharanthus and serpentwood protein sequences used in this project was obtained from the MPGR database (http://medicinalplantgenomics.msu.edu/index.shtml). Sequences for Arabidopsis proteins were obtained from TAIR10 (http://www.arabidopsis.org/). Protein sequences from Amborella (http://www.amborella.org/), bladderwort (http://genomevolution.org/CoGe/OrganismView.pl?oid= 36222), pepper (http://peppergenome.snu.ac.kr/), and rice (http://rice.plantbiology.msu.edu/) and were obtained from their respective databases. Tomato and potato protein sequences were obtained from the Sol Genomics Network database (http://solgenomics.net/). Sequence alignments and phylogenetic trees generated by this study were deposited into TreeBase under study identification number 15861 (http://purl.org/phylo/treebase/phylows/study/ TB2:S15861).

\section{Additional files}

Additional file 1: Table S1. The list of WRKY TFs present or absent from the Affymetrix arrays used in this study. The Arabidopsis Affymetrix array contains probes to identify the expression of 61 WRKY TFs. Eleven of the 72 WRKY TFs in Arabidopsis are not represented on the array.

Additional file 2: Table S2. The WRKY TFs identified as having significantly altered gene expression in at least one jasmonate treated dataset. WRKYs cited as identified in this study are those which were had significantly altered gene expression and survived the B-H FDR in at least one dataset. N/A indicates a probe to identify that WRKY is not available on the Affymetrix array but has been reported to be involved in jasmonate response. References are shown for WRKYs with reported function in jasmonate response.

Additional file 3: Table S3. The fold change of jasmonate responsive Arabidopsis WRKY TFs from five microarray datasets. Only those jasmonate responsive AtWRKYs which survived application of the $\mathrm{B}-\mathrm{H}$ FDR are included.

Additional file 4: Table S4. Arabidopsis WRKY TFs were analyzed for differential expression by genotype in A) coil and B) myc2 mutants or C) by time. Analysis was performed using a two-way ANOVA. WRKYS before and after the application of the B-H FDR are presented.

Additional file 5: Figure S1. Hierarchical cluster analysis of the Arabidopsis WRKY TF family was performed using GenePattern. The clustering method was a pairwise average linkage with distance measured using the Pearson correlation coefficient. Data was log transformed. The median value was subtracted from each row. Color is based on global expression with purple being up-regulated and green down-regulated.

Additional file 6: Table S5. WRKY domain containing proteins were identified using 4 sources: manual searching, PlantTFcat, NCBI CDD, and MPGR. Rows indicate overlap in genes identified from the different sources. The bottom row provides a total number of genes identified by each method.

Additional file 7: Figure S2. A phylogenetic tree constructed with nine plant species. The species tree was computed using the NCBI Common Tree then visualized with MEGA5 software.

Additional file 8: Figure S3. The phylogenetic tree with $A$. thaliana (green square), A. trichopoda (purple triangle), C. annuum (teal dot), $O$. sativa (blue diamond), R. serpentina (red dot), and U. gibba (gold square) 
was constructed in MEGA5 using the Neighbor-Joining method with P-distance substitution model and a bootstrap value of 2000. Proteins used as an outgroup are indicated by a black triangle. WRKY domain alignment was performed with ClustalW.

Additional file 9: Figure S4. The phylogenetic tree with A. thaliana (green square), A. trichopoda (purple triangle), O. sativa (blue diamond), S. lycopersicum (gold square), and S. tuberosum (red dot) was constructed in MEGA5 using the Neighbor-Joining method with P-distance substitution model and a bootstrap value of 2000 . Proteins used as an outgroup are indicated by a black triangle. WRKY domain alignment was performed with ClustalW.

Additional file 10: Table S6. Catharanthus contained at least 48 WRKY TFs with 56 WRKY domains. The 70 amino acid sequence of each WRKY domain is provided.

Additional file 11: Figure S5. A-B. A. The phylogenetic relationship and alignment of CrWRKY1 to other group III WRKY TFs. The phylogenetic tree was constructed in MEGA5 using the Neighbor-Joining method with P-distance substitution model and a bootstrap value of 2000. WRKY domain alignment was performed with ClustalW. B. Alignment of the closest related rice WRKY genes and Catharanthus group III WRKYs was performed using ClustalW.

Additional file 12: Table S7. A list of primers used in GRT-PCR to measure transcript levels.

Additional file 13: Table S8. A list or primers used in cloning to isolate full WRKY domains for those TFs in the Catharanthus MPGR database with partial domain sequences.

\section{Abbreviations}

MPGR: Medicinal Plant Genomics Resource; PCR: Polymerase chain reaction; qRT-PCR: Quantitative reverse-transcription polymerase chain reaction; TF: Transcription factor; TIA: Terpene indole alkaloid.

\section{Competing interest}

The authors declare that they have no competing interests.

\section{Authors' contributions}

LY conceived and directed the project. CS planned and designed the project. Statistical and phylogenetic analyses were performed by CS. CS, SP, and BP assisted in cloning incomplete WRKY fragments and GRT-PCR analysis. CS, SP and LY wrote the manuscript. All authors have read and approved of the manuscript.

\section{Acknowledgements}

The authors would like to thank K. Shen (University of Kentucky) for providing editorial comments and corrections to the manuscript. Funding for C. Schluttenhofer and this work was provided by the Plant and Soil Sciences Department, University of Kentucky.

Received: 4 October 2013 Accepted: 13 June 2014 Published: 20 June 2014

\section{References}

1. Junker RR, Blüthgen $\mathrm{N}$ : Floral scents repel facultative flower visitors, but attract obligate ones. Ann Bot 2010, 105(5):777-782.

2. Muroi A, Ramadan A, Nishihara M, Yamamoto M, Ozawa R, Takabayashi J, Arimura G-i: The composite effect of transgenic plant volatiles for acquired immunity to herbivory caused by inter-plant communications. PLoS One 2011, 6(10):e24594.

3. Roepke J, Salim V, Wu M, Thamm AMK, Murata J, Ploss K, Boland W, De Luca V: Vinca drug components accumulate exclusively in leaf exudates of Madagascar periwinkle. Proc Natl Acad Sci 2010, 107(34):15287-15292.

4. Holland JF, Scharlau C, Gailani S, Krant MJ, Olson KB, Horton J, Shnider BI, Lynch JJ, Owens A, Carbone PP, Colsky J, Grob D, Miller SP, Hall TC: Vincristine treatment of advanced cancer: a cooperative study of 392 cases. Cancer Res 1973, 33(6):1258-1264.

5. Binder BYK, Peebles CAM, Shanks JV, San K-Y: The effects of UV-B stress on the production of terpenoid indole alkaloids in Catharanthus roseus hairy roots. Biotechnol Prog 2009, 25(3):861-865.
6. Ramani S, Chelliah J: UV-B-induced signaling events leading to enhancedproduction of catharanthine in Catharanthus roseus cell suspension cultures. BMC Plant Biol 2007, 7(1):61.

7. Menke FLH, Parchmann S, Mueller MJ, Kijne JW, Memelink J: Involvement of the Octadecanoid pathway and protein phosphorylation in fungal elicitor-induced expression of terpenoid indole alkaloid biosynthetic genes in Catharanthus roseus. Plant Physiol 1999, 119(4):1289-1296.

8. Frischknecht PM, Bättig M, Baumann TW: Effect of drought and wounding stress on indole alkaloid formation in Catharanthus roseus. Phytochemistry 1987, 26(3):707-710.

9. Vázquez-Flota F, Carrillo-Pech M, Minero-García Y, de Lourdes M-HM: Alkaloid metabolism in wounded Catharanthus roseus seedlings. Plant Physiol Biochem 2004, 42(7-8):623-628.

10. Jaleel CA, Manivannan P, Kishorekumar A, Sankar B, Gopi R, Somasundaram $R$, Panneerselvam R: Alterations in osmoregulation, antioxidant enzymes and indole alkaloid levels in Catharanthus roseus exposed to water deficit. Colloids Surf B Biointerfaces 2007, 59(2):150-157.

11. Dutta A, Sen J, Deswal R: Downregulation of terpenoid indole alkaloid biosynthetic pathway by low temperature and cloning of a AP2 type C-repeat binding factor (CBF) from Catharanthus roseus (L) G Don. Plant Cell Rep 2007, 26(10):1869-1878.

12. Dutta A, Sen J, Deswal R: New evidences about strictosidine synthase (Str) regulation by salinity, cold stress and nitric oxide in Catharanthus roseus. $J$ Plant Biochem Biotechnol 2013, 22(1):124-131.

13. Wei S: Methyl jasmonic acid induced expression pattern of terpenoid indole alkaloid pathway genes in Catharanthus roseus seedlings. Plant Growth Regul 2010, 61(3):243-251.

14. Van Der Fits L, Memelink J: The jasmonate-inducible AP2/ERF-domain transcription factor ORCA3 activates gene expression via interaction with a jasmonate-responsive promoter element. Plant J 2001, 25(1):43-53.

15. Menke FLH, Champion A, Kijne JW, Memelink J: A novel jasmonate- and elicitor-responsive element in the periwinkle secondary metabolite biosynthetic gene Str interacts with a jasmonate- and elicitor-inducible AP2-domain transcription factor, ORCA2. EMBO J 1999, 18(16):4455-4463.

16. Zhang H, Hedhili S, Montiel G, Zhang Y, Chatel G, Pré M, Gantet P, Memelink J: The basic helix-loop-helix transcription factor CrMYC2 controls the jasmonate-responsive expression of the ORCA genes that regulate alkaloid biosynthesis in Catharanthus roseus. Plant J 2011, 67(1):61-71.

17. Sibéril Y, Benhamron S, Memelink J, Giglioli-Guivarc'h N, Thiersault M, Boisson B, Doireau P, Gantet P: Catharanthus roseus G-box binding factors 1 and 2 act as repressors of strictosidine synthase gene expression in cell cultures. Plant Mol Biol 2001, 45(4):477-488.

18. Pauw B, Hilliou FAO, Martin VS, Chatel G, de Wolf CJF, Champion A, Pré M, van Duijn B, Kijne JW, van der Fits L, Memelink J: Zinc finger proteins act as transcriptional repressors of Alkaloid Biosynthesis genes in Catharanthus roseus. J Biol Chem 2004, 279(51):52940-52948.

19. Ren X, Chen Z, Liu Y, Zhang H, Zhang M, Liu Q, Hong X, Zhu J-K, Gong Z: $\mathrm{ABO}$, a WRKY transcription factor, mediates plant responses to abscisic acid and drought tolerance in Arabidopsis. Plant J 2010, 63(3):417-429.

20. Jiang Y, Deyholos M: Functional characterization of Arabidopsis $\mathrm{NaCl}$ inducible WRKY25 and WRKY33 transcription factors in abiotic stresses. Plant Mol Biol 2009, 69(1-2):91-105.

21. Devaiah BN, Karthikeyan AS, Raghothama KG: WRKY75 Transcription factor is a modulator of phosphate acquisition and root development in Arabidopsis. Plant Physiol 2007, 143(4):1789-1801.

22. Chen L, Zhang L, Yu D: Wounding-Induced WRKY 8 Is Involved in Basal Defense in Arabidopsis. Mol Plant Microbe Interact 2010, 23(5):558-565.

23. Zou C, Jiang W, Yu D: Male gametophyte-specific WRKY34 transcription factor mediates cold sensitivity of mature pollen in Arabidopsis. J Exp Bot 2010, 61(14):3901-3914.

24. Li S, Zhou X, Chen L, Huang W, Yu D: Functional characterization of Arabidopsis thaliana WRKY39 in heat stress. Mol Cells 2010, 29(5):475-483.

25. Li S, Fu Q, Chen L, Huang W, Yu D: Arabidopsis thaliana WRKY25, WRKY26, and WRKY33 coordinate induction of plant thermotolerance. Planta 2011, 233(6):1237-1252.

26. Skibbe M, Qu N, Galis I, Baldwin IT: induced plant defenses in the natural environment: Nicotiana attenuata WRKY3 and WRKY6 coordinate responses to herbivory. Plant Cell 2008, 20(7):1984-2000.

27. Zheng Z, Qamar SA, Chen Z, Mengiste T: Arabidopsis WRKY33 transcription factor is required for resistance to necrotrophic fungal pathogens. Plant J 2006, 48(4):592-605. 
28. Wang $H$, Hao J, Chen $X$, Hao Z, Wang $X$, Lou $Y$, Peng $Y$, Guo Z: Overexpression of rice WRKY89 enhances ultraviolet $B$ tolerance and disease resistance in rice plants. Plant Mol Biol 2007, 65(6):799-815

29. Zhang $Y$, Wang $L$ : The WRKY transcription factor superfamily: its origin in eukaryotes and expansion in plants. BMC Evol Biol 2005, 5(1):1

30. Machens F, Becker M, Umrath F, Hehl R: Identification of a novel type of WRKY transcription factor binding site in elicitor-responsive cis sequences from Arabidopsis thaliana. Plant Mol Biol 2014, 84(4-5):371-385.

31. Brand LH, Fischer NM, Harter K, Kohlbacher O, Wanke D: Elucidating the evolutionary conserved DNA-binding specificities of WRKY transcription factors by molecular dynamics and in vitro binding assays. Nucleic Acids Res 2013, 41(21):9764-9778.

32. Eulgem T, Rushton PJ, Robatzek S, Somssich IE: The WRKY superfamily of plant transcription factors. Trends Plant Sci 2000, 5(5):199-206.

33. Dong J, Chen C, Chen Z: Expression profiles of the Arabidopsis WRKY gene superfamily during plant defense response. Plant Mol Biol 2003, 51(1):21-37.

34. Kalde M, Barth M, Somssich IE, Lippok B: Members of the Arabidopsis WRKY Group III Transcription Factors Are Part of Different Plant Defense Signaling Pathways. Mol Plant Microbe Interact 2003, 16(4):295-305

35. Yu D, Chen C, Chen Z: Evidence for an important role of WRKY DNA binding proteins in the regulation of NPR1 gene expression. Plant Cell 2001, 13(7):1527-1540.

36. Li J, Brader G, Palva ET: The WRKY70 transcription factor: a node of convergence for Jasmonate-Mediated and Salicylate-Mediated signals in plant defense. Plant Cell 2004, 16(2):319-331.

37. Birkenbihl RP, Diezel C, Somssich IE: Arabidopsis WRKY33 is a key transcriptional regulator of Hormonal and Metabolic Responses toward Botrytis cinerea infection. Plant Physiol 2012, 159(1):266-285.

38. Pandey SP, Roccaro M, Schön M, Logemann E, Somssich IE: Transcriptional reprogramming regulated by WRKY18 and WRKY40 facilitates powdery mildew infection of Arabidopsis. Plant J 2010, 64(6):912-923.

39. Zheng Z, Mosher S, Fan B, Klessig D, Chen Z: Functional analysis of Arabidopsis WRKY25 transcription factor in plant defense against Pseudomonas syringae. BMC Plant Biol 2007, 7(1):2

40. Journot-Catalino N, Somssich IE, Roby D, Kroj T: The transcription factors WRKY11 and WRKY17 act as negative regulators of basal resistance in Arabidopsis thaliana. Plant Cell 2006, 18(11):3289-3302.

41. L-t W, Zhong G-m, Wang J-m, Li X-f, Song X, Yang Y: Arabidopsis WRKY28 transcription factor is required for resistance to necrotrophic pathogen, Botrytis cinerea. Afr J Microbiol Res 2011, 5(30):5481-5488.

42. Robatzek S, Somssich IE: A new member of the Arabidopsis WRKY transcription factor family, AtWRKY6, is associated with both senescence- and defence-related processes. Plant J 2001, 28(2):123-133.

43. Hu Y, Dong Q, Yu D: Arabidopsis WRKY46 coordinates with WRKY70 and WRKY53 in basal resistance against pathogen Pseudomonas syringae. Plant Sci 2012, 185:288-297.

44. Zhou C, Zhang L, Duan J, Miki B, Wu K: HISTONE DEACETYLASE19 Is Involved in Jasmonic Acid and Ethylene signaling of pathogen response in Arabidopsis. Plant Cell 2005, 17(4):1196-1204.

45. Kim K-C, Lai Z, Fan B, Chen Z: Arabidopsis WRKY38 and WRKY62 transcription factors interact with Histone Deacetylase 19 in Basal Defense. Plant Cell 2008, 20(9):2357-2371.

46. Suttipanta N, Pattanaik S, Kulshrestha M, Patra B, Singh SK, Yuan L: The transcription factor CrWRKY1 positively regulates the Terpenoid Indole Alkaloid Biosynthesis in Catharanthus roseus. Plant Physiol 2011, 157(4):2081-2093.

47. Li S, Zhang P, Zhang M, Fu C, Yu L: Functional analysis of a WRKY transcription factor involved in transcriptional activation of the DBAT gene in Taxus chinensis. Plant Biol 2013, 15(1):19-26.

48. Kato N, Dubouzet E, Kokabu Y, Yoshida S, Taniguchi Y, Dubouzet JG, Yazak $K$, Sato F: Identification of a WRKY protein as a transcriptional regulator of Benzylisoquinoline Alkaloid Biosynthesis in Coptis japonica. Plant Cell Physiol 2007, 48(1):8-18.

49. Xu Y-H, Wang J-W, Wang S, Wang J-Y, Chen X-Y: Characterization of GaWRKY1, a cotton transcription factor that regulates the Sesquiterpene Synthase Gene (+)- $\delta$-Cadinene Synthase-A. Plant Physiol 2004, 135(1):507-515.

50. Ma D, Pu G, Lei C, Ma L, Wang H, Guo Y, Chen J, Du Z, Wang H, Li G, Ye H, Liu B: Isolation and characterization of AaWRKY1, an Artemisia annua transcription factor that regulates the Amorpha-4,11-diene synthase gene, a key gene of Artemisinin Biosynthesis. Plant Cell Physiol 2009, 50(12):2146-2161

51. Zhang Q, Zhu J, Ni Y, Cai Y, Zhang Z: Expression profiling of HbWRKY1, an ethephon-induced WRKY gene in latex from Hevea brasiliensis in responding to wounding and drought. Trees 2012, 26(2):587-595.

52. Mao G, Meng X, Liu Y, Zheng Z, Chen Z, Zhang S: Phosphorylation of a WRKY transcription factor by two pathogen-responsive MAPKs Drives Phytoalexin Biosynthesis in Arabidopsis. Plant Cell 2011, 23(4):1639-1653.

53. Sun Y, Niu Y, Xu J, Li Y, Luo H, Zhu Y, Liu M, Wu Q, Song J, Sun C, Chen S: Discovery of WRKY transcription factors through transcriptome analysis and characterization of a novel methyl jasmonate-inducible PqWRKY1 gene from Panax quinquefolius. Plant Cell Tiss Org Cult 2013, 114:269-277.

54. Sheard LB, Tan X, Mao H, Withers J, Ben-Nissan G, Hinds TR, Kobayashi Y, Hsu F-F, Sharon M, Browse J, He SY, Rizo J, Howe GA, Zheng N: Jasmonate perception by inositol-phosphate-potentiated COI1-JAZ co-receptor. Nature 2010, 468(7322):400-405.

55. Xie D-X, Feys BF, James S, Nieto-Rostro M, Turner JG: COI1: an Arabidopsis gene required for Jasmonate-regulated defense and fertility. Science 1998, 280(5366):1091-1094.

56. Dombrecht B, Xue GP, Sprague SJ, Kirkegaard JA, Ross JJ, Reid JB, Fitt GP, Sewelam N, Schenk PM, Manners JM, Kazan K: MYC2 differentially modulates diverse Jasmonate-Dependent functions in Arabidopsis. Plant Cell 2007, 19(7):2225-2245.

57. Lorenzo O, Chico JM, Sanchez-Serrano JJ, Solano R: JASMONATE-INSENSITIVE1 Encodes a MYC Transcription Factor Essential to Discriminate between different Jasmonate-Regulated defense responses in Arabidopsis. Plant Cell 2004, 16(7):1938-1950.

58. Eisen MB, Spellman PT, Brown PO, Botstein D: Cluster analysis and display of genome-wide expression patterns. Proc Natl Acad Sci 1998, 95(25):14863-14868.

59. Góngora-Castillo E, Childs KL, Fedewa G, Hamilton JP, Liscombe DK, Magallanes-Lundback M, Mandadi KK, Nims E, Runguphan W, Vaillancourt B, Varbanova-Herde M, Dellapenna D, McKnight TD, O'Connor S, Buell CR: Development of transcriptomic resources for interrogating the Biosynthesis of Monoterpene Indole Alkaloids in medicinal plant species. PLoS One 2012, 7(12):e52506.

60. Marchler-Bauer A, Lu S, Anderson JB, Chitsaz F, Derbyshire MK, DeWeese-Scott C, Fong JH, Geer LY, Geer RC, Gonzales NR, Gwadz M, Hurwitz DI, Jackson JD, Ke Z, Lanczycki CJ, Lu F, Marchler GH, Mullokandov M, Omelchenko MV, Robertson CL, Song JS, Thanki N, Yamashita RA, Zhang D, Zhang N, Zheng C, Bryant SH: CDD: a Conserved Domain Database for the functional annotation of proteins. Nucleic Acids Res 2011, 39(suppl 1):D225-D229.

61. Li H-L, Zhang L-B, Guo D, Li C-Z, Peng S-Q: Identification and expression profiles of the WRKY transcription factor family in Ricinus communis. Gene 2012, 503(2):248-253.

62. Rushton PJ, Somssich IE, Ringler P, Shen QJ: WRKY transcription factors. Trends Plant Sci 2010, 15(5):247-258

63. Rensing SA, Lang D, Zimmer AD, Terry A, Salamov A, Shapiro H, Nishiyama T, Perroud P-F, Lindquist EA, Kamisugi Y, Tanahashi T, Sakakibara K, Fujita T, Oishi K, Shin-I T, Kuroki Y, Toyoda A, Suzuki Y, Hashimoto S, Yamaguchi K, Sugano S, Kohara Y, Fujiyama A, Anterola A, Aoki S, Ashton N, Barbazuk WB, Barker E, Bennetzen JL, Blankenship R, et al: The Physcomitrella genome reveals evolutionary insights into the conquest of land by plants. Science 2008, 319(5859):64-69.

64. Ling J, Jiang W, Zhang Y, Yu H, Mao Z, Gu X, Huang S, Xie B: Genome-wide analysis of WRKY gene family in Cucumis sativus. BMC Genomics 2011, 12(1):471.

65. Miao L-X, Zhang Y-c, Yang X-f, Jiang G-h: Genome-wide identification and analysis of WRKY transcription factors in Fragaria vesca. J Nucl Agriculture Sci 2012, 8:1124-1131.

66. Pandey SP, Somssich IE: The role of WRKY transcription factors in plant immunity. Plant Physiol 2009, 150(4):1648-1655.

67. Xiong W, Xu X, Zhang L, Wu P, Chen Y, Li M, Jiang H, Wu G: Genome-wide analysis of the WRKY gene family in physic nut (Jatropha curcas L.). Gene 2013, 524(2):124-132.

68. The Tomato Genome Consortium: The tomato genome sequence provides insights into fleshy fruit evolution. Nature 2012, 485(7400):635-641.

69. The Potato Genome Sequencing Consortium: Genome sequence and analysis of the tuber crop potato. Nature 2011, 475(7355):189-195.

70. Kim S, Park M, Yeom SI, Kim YM, Lee JM, Lee HA, Seo E, Choi J, Cheong K, Kim KT, Jung K, Lee GW, Oh SK, Bae C, Kim SB, Lee HY, Kim SY, Kim MS, Kang BC, Jo YD, Yang HB, Jeong HJ, Kang WH, Kwon JK, Shin C, Lim JY, Park 
$J H$, Huh JH, Kim JS, Kim BD, et al: Genome sequence of the hot pepper provides insights into the evolution of pungency in Capsicum species. Nat Genet 2014, 46(3):270-278.

71. Ibarra-Laclette E, Lyons E, Hernandez-Guzman G, Perez-Torres CA, CarreteroPaulet L, Chang T-H, Lan T, Welch AJ, Juarez MJA, Simpson J, FernándezCortés A, Arteaga-Vázquez M, Góngora-Castillo E, Acevedo-Hernández G, Schuster SC, Himmelbauer H, Minoche AE, Xu S, Lynch M, Oropeza-Aburto A, Cervantes-Pérez SA, de Jesús Ortega-Estrada M, Cervantes-Luevano Jl, Michael TP, Mockler T, Bryant D, Herrera-Estrella A, Albert VA, Herrera-Estrella L: Architecture and evolution of a minute plant genome. Nature 2013, 498(7452):94-98

72. Huang S, Gao Y, Liu J, Peng X, Niu X, Fei Z, Cao S, Liu Y: Genome-wide analysis of WRKY transcription factors in Solanum lycopersicum. Mol Genet Genomics 2012, 287(6):495-513

73. Wu K-L, Guo Z-J, Wang H-H, Li J: The WRKY family of transcription factors in rice and Arabidopsis and their origins. DNA Res 2005, 12(1):9-26.

74. Tripathi P, Rabara R, Langum T, Boken A, Rushton D, Boomsma D, Rinerson C, Rabara J, Reese R, Chen X, Rohila JS, Rushton PJ: The WRKY transcription factor family in Brachypodium distachyon. BMC Genomics 2012, 13(1):270.

75. Wei K-F, Chen J, Chen Y-F, Wu L-J, Xie D-X: Molecular phylogenetic and expression analysis of the complete WRKY transcription factor family in Maize. DNA Res 2012, 19(2):153-164.

76. Amborella Genome Project: The Amborella genome and the evolution of flowering plants. Science 2013, 342(6165):1241089.

77. Li L, Stoeckert CJ, Roos DS: OrthoMCL: identification of Ortholog groups for eukaryotic genomes. Genome Res 2003, 13(9):2178-2189.

78. Duan M-R, Nan J, Liang Y-H, Mao P, Lu L, Li L, Wei C, Lai L, Li Y, Su X-D: DNA binding mechanism revealed by high resolution crystal structure of Arabidopsis thaliana WRKY1 protein. Nucleic Acids Res 2007, 35(4):1145-1154.

79. van Verk MC, Pappaioannou D, Neeleman L, Bol JF, Linthorst HJM: A Novel WRKY Transcription Factor Is Required for Induction of PR-1a gene expression by Salicylic Acid and Bacterial Elicitors. Plant Physiol 2008, 146(4):1983-1995.

80. Oh S-K, Baek K-H, Park JM, Yi SY, Yu SH, Kamoun S, Choi D: Capsicum annuum WRKY protein CaWRKY1 is a negative regulator of pathogen defense. New Phytol 2008, 177(4):977-989.

81. Sun C, Palmqvist S, Olsson H, Borén M, Ahlandsberg S, Jansson C: A Novel WRKY Transcription Factor, SUSIBA2, participates in sugar signaling in Barley by binding to the sugar-responsive elements of the iso 1 promoter. Plant Cell Online 2003, 15(9):2076-2092.

82. Aerts RJ, Gisi D, De Carolis E, De Luca V, Baumann TW: Methyl jasmonate vapor increases the developmentally controlled synthesis of alkaloids in Catharanthus and Cinchona seedlings. Plant J 1994, 5(5):635-643.

83. Zabetakis I, Edwards R, O'Hagan D: Elicitation of tropane alkaloid biosynthesis in transformed root cultures of Datura stramonium. Phytochemistry 1999, 50(1):53-56.

84. Biondi S, Fornalé S, Oksman-Caldentey KM, Eeva M, Agostani S, Bagni N: Jasmonates induce over-accumulation of methylputrescine and conjugated polyamines in Hyoscyamus muticus L. root cultures. Plant Cell Rep 2000, 19(7):691-697.

85. Yu K-W, Gao W-Y, Son S-H, Paek K-Y: Improvement of ginsenoside production by jasmonic acid and some other elicitors in hairy root culture of ginseng (Panax ginseng C. A. Meyer). In Vitr Cell Dev Biol Plant 2000, 36(5):424-428.

86. Collu G, Unver N, Peltenburg-Looman AMG, van der Heijden R, Verpoorte R, Memelink J: Geraniol 10-hydroxylase, a cytochrome P450 enzyme involved in terpenoid indole alkaloid biosynthesis. FEBS Lett 2001, 508(2):215-220.

87. Van der Fits L, Memelink J: ORCA3, a Jasmonate-Responsive transcriptional regulator of plant primary and secondary metabolism. Science 2000, 289(5477):295-297.

88. Peebles CAM, Hughes EH, Shanks JV, San K-Y: Transcriptional response of the terpenoid indole alkaloid pathway to the overexpression of ORCA3 along with jasmonic acid elicitation of Catharanthus roseus hairy roots over time. Metab Eng 2009, 11(2):76-86.

89. Suttipanta N, Pattanaik S, Gunjan S, Xie CH, Littleton J, Yuan L: Promoter analysis of the Catharanthus roseus geraniol 10-hydroxylase gene involved in terpenoid indole alkaloid biosynthesis. Biochim Biophys Acta (BBA) Gene Struct Expr 2007, 1769(2):139-148.

90. Pan Q, Chen Y, Wang Q, Yuan F, Xing S, Tian Y, Zhao J, Sun X, Tang K: Effect of plant growth regulators on the biosynthesis of vinblastine, vindoline and catharanthine in Catharanthus roseus. Plant Growth Regul 2010, 60(2):133-141.

91. Vanderauwera S, Zimmermann P, Rombauts S, Vandenabeele S, Langebartels C, Gruissem W, Inzé D, Van Breusegem F: Genome-wide analysis of hydrogen peroxide-regulated gene expression in Arabidopsis reveals a high light-induced transcriptional cluster involved in Anthocyanin Biosynthesis. Plant Physiol 2005, 139(2):806-821.

92. Geu-Flores F, Sherden NH, Courdavault V, Burlat V, Glenn WS, Wu C, Nims E,

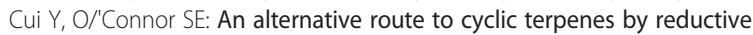
cyclization in iridoid biosynthesis. Nature 2012, 492(7427):138-142.

93. Raina S, Wankhede D, Jaggi M, Singh P, Jalmi S, Raghiram B, Sheikh A, Sinha A: CrMPK3, a mitogen activated protein kinase from Catharanthus roseus and its possible role in stress induced biosynthesis of monoterpenoid indole alkaloids. BMC Plant Biol 2012, 12(1):134.

94. Ayora-Talavera T, Chappell J, Lozoya-Gloria E, Loyola-Vargas V: Overexpression in Catharanthus roseus hairy roots of a truncated hamster 3-hydroxy-3methylglutaryl-CoA reductase gene. Appl Biochem Biotechnol 2002, 97(2):135-145.

95. Chen H, Lai Z, Shi J, Xiao Y, Chen Z, Xu X: Roles of arabidopsis WRKY18, WRKY40 and WRKY60 transcription factors in plant responses to abscisic acid and abiotic stress. BMC Plant Biol 2010, 10(1):281.

96. Jaleel CA, Sankar B, Sridharan R, Panneerselvam R: Soil salinity alters growth, chlorophyll content, and secondary metabolite accumulation in Catharanthus roseus. Turk J Biol 2008, 32:79-83.

97. Shoji T, Hashimoto T: Tobacco MYC2 Regulates Jasmonate-Inducible Nicotine Biosynthesis Genes Directly and By Way of the NIC2-Locus ERF Genes. Plant Cell Physiol 2011, 52(6):1117-1130.

98. Montiel G, Zarei A, Körbes AP, Memelink J: The Jasmonate-Responsive element from the ORCA3 promoter from Catharanthus roseus is active in Arabidopsis and is controlled by the transcription factor AtMYC2. Plant Cell Physiol 2011, 52(3):578-587.

99. Bolstad BM, Irizarry RA, Åstrand M, Speed TP: A comparison of normalization methods for high density oligonucleotide array data based on variance and bias. Bioinformatics 2003, 19(2):185-193.

100. Saeed A, Sharov V, White J, Li J, Liang W, Bhagabati N, Braisted J, Klapa M, Currier T, Thiagarajan M: TM4: a free, open-source system for microarray data management and analysis. Biotechniques 2003, 34(2):374.

doi:10.1186/1471-2164-15-502

Cite this article as: Schluttenhofer et al:: Analyses of Catharanthus roseus and Arabidopsis thaliana WRKY transcription factors reveal involvement in jasmonate signaling. BMC Genomics 2014 15:502.

\section{Submit your next manuscript to BioMed Central and take full advantage of:}

- Convenient online submission

- Thorough peer review

- No space constraints or color figure charges

- Immediate publication on acceptance

- Inclusion in PubMed, CAS, Scopus and Google Scholar

- Research which is freely available for redistribution

Submit your manuscript at www.biomedcentral.com/submit
C Biomed Central 